\title{
Deep penetration of ultra-high molecular weight polyethylene composites by a sharp- tipped punch
}

\author{
B.G. Liu ${ }^{\mathrm{a}}$, K. Kandan ${ }^{\mathrm{a}, \mathrm{b}}$, H.N.G. Wadley ${ }^{\mathrm{c}}$ and V.S. Deshpande ${ }^{\mathrm{a}^{*}}$ \\ a Department of Engineering, University of Cambridge, \\ Trumpington Street, Cambridge CB2 1PZ, UK. \\ ${ }^{\mathrm{b}}$ School of Engineering, De Montfort University, \\ The Gateway, Leicester LE1 9BH, UK. \\ ${ }^{\mathrm{c}}$ Department of Material Science \& Engineering, School of Engineering and Applied Science, \\ University of Virginia, Charlottesville, VA 22904, USA.
}

\begin{abstract}
The penetration of unidirectional (UD) and $\left[0^{\circ} / 90^{\circ}\right]$ cross-ply ultra-high molecular weight polyethylene fibre composites by sharp-tipped cylindrical punches has been investigated. While the measured penetration pressure for both composite types increased with decreasing punch diameter, the pressure was significantly higher for the cross-ply composites and increased with decreasing ply thickness. A combination of optical microscopy and X-ray tomography revealed that in both composites, the sharp-tipped punch penetrated without fibre fracture by the formation of mode-I cracks along the fibre directions, followed by the wedging open of the crack by the advancing punch. In the cross-ply composites, delamination between adjacent $0^{\circ}$ and $90^{\circ}$ plies also occurred to accommodate the incompatible deformation between plies containing orthogonal mode-I cracks. Micromechanical models for the steadystate penetration pressure were developed for both composites. To account for material anisotropy as well as the large shear strains and fibre rotations, the deformation of the composites was modelled via a pressure-dependent crystal plasticity framework. Intra and inter-ply fracture were accounted for via mode-I and delamination toughnesses respectively. These models account for the competition between deformation and fracture of the plies and accurately predict the measured steady-state penetration pressures over the wide range of punch diameters and ply thicknesses investigated here. Design maps for the penetration resistance of cross-ply composites were constructed using these models and subsequently used to infer composite designs that maximise the penetration resistance for a user prescribed value of fibre strength.
\end{abstract}

Keywords: deep penetration; fracture mechanics; composites; delamination

*Corresponding author. E-mail address: vsd@eng.cam.ac.uk. 


\section{Introduction}

There is considerable interest in the use of ultra-high molecular weight polyethylene (UHMWPE) fibre composites in ballistic protection applications. This interest has primarily been driven by the high strength and stiffness to weight ratios of the polyethylene fibres (Van Dingenen, 1989; Russell et al., 2013). These high specific properties also imply that UHMWPE composites have found application in ropes, sails and tear resistant fabrics. For ballistic applications, $10-20 \mu \mathrm{m}$ diameter fibres are combined with thermoplastic matrices to form unidirectional plies with ply thicknesses ranging from $20 \mu \mathrm{m}$ to $100 \mu \mathrm{m}$ and fibre volume fractions of $80-85 \%$ with the unidirectional plies typically combined to form a $\left[0^{\circ} / 90^{\circ}\right]$ crossply composite. Examples of such composites include Dyneema ${ }^{\circledR}$ manufactured by DSM (DSM, Het Overloon 1, 6411 TE Heerlen, The Netherlands) and Spectra ${ }^{\circledR}$ marketed by Honeywell (Honeywell Advanced Fibers and Composites, Morris Township, N.J., USA).

A number of studies have reported the static and dynamic response of UHMWPE fibres and composites (Karthikeyan et al., 2013; Govaert and Peijs, 1995; Wilding and Ward, 1978). For example, Russell et al. (2013) observed that UHMWPE composites have tensile strengths of a few GPa but a shear strength of only a few MPa. Moreover, they found that the tensile strength of UHMWPE fibres displays nearly no strain rate dependence for strain rates up to $10^{3} \mathrm{~s}^{-1}$. These studies have been accompanied by efforts to understand the deformation and failure mechanisms of the UHMWPE composites under both quasi-static and dynamic loading. However, the studies to-date have been largely restricted to the out-of-plane (transverse) loading of the composites by blunt projectiles/indenters. Early theoretical investigations by Phoenix and Porwall (2003) of the transverse impact of UHMWPE composite plates suggested that the failure of these composites occurs in a membrane stretching mode with the Cunniff (1999) dimensional analysis providing a reasonable estimate of the material properties that set the ballistic limit. For this membrane stretching approximation, the composite plate either completely fails or suffers no fracture. However, a series of more recent experimental studies using flat-bottomed (O'Masta et al., 2016; Attwood et al., 2016;) and spherical (O'Masta et al. 2015; Heisserer et al., 2013; Karthikeyan and Russel, 2014) projectiles revealed that penetration occurs in a progressive manner in which fibre failure occurring by the indirect tension mechanism introduced by Attwood et al. (2014). These studies indicate the penetration response is sensitive to the shear strength of the composite with the penetration mode eventually transitioning to a shear-off mode with increasing shear strength (Cheeseman and Bogetti, 2003; Attwood et al., 2016). In parallel with these experimental investigations of the penetration mechanisms, significant efforts have sought to model the observed responses. Iannucci and Pope (2011) modelled the cross-ply composites as a homogenized medium with a non-linear shear stress versus strain relationship fitted to measured data. By contrast, Grujicic et al. (2008) proposed a multi-scale material model for a cross-ply UHMWPE composite. They attempted to use fundamental material properties of the fibres and the matrix within a homogenisation framework. While both models give results that are in qualitative agreement with measurements, they do not predict the observed progressive mode of penetration with sufficient accuracy.

While the investigations of the penetration resistance of UHMWPE composites described above have largely been restricted to blunt projectiles, the effect of the projectile tip shape on the penetration resistance of traditional carbon and glass fibre composites has received extensive attention. Experimental investigations have ranged from low-velocity drop weight tests (Mitrevski et al., 2005) to high-velocity gas gun tests (Mines et al., 1999; Onyechi et al., 2014) with flat, conical and hemispherical tipped projectiles. While pervasive damage in these brittle composites made the effect of the projectile tip shape on the penetration mode difficult 
to quantify, clear differences in the energy absorption and ballistic limit were observed. Modelling efforts for these materials have been predominantly based on continuum damage formulations with both analytical (Wen, 2000) and numerical (Kim and Goo, 1997) approaches. A related class of investigations is the stab/puncture resistance of composites performed with the aim of developing composites for application in stab-resistant or kniferesistant vests; readers are referred to Bilisik (2017) for a recent review. These studies are restricted to the quasi-static penetration by sharp-tipped punches of composites that include woven aramid fabrics (Mayo et al., 2009; Decker et al., 2007), Dyneema ${ }^{\circledR}$ (Li et al., 2016) and non-woven mats (Bao et al., 2016). These investigations illustrate the importance of matrix properties in setting the penetration resistance, but a quantitative understanding of the effect of material properties upon penetration by sharp penetrators is missing. This in large part is due to the absence of a modelling framework.

In this study, we report a combined experimental and theoretical investigation of the penetration resistance of unidirectional and cross-ply UHMWPE fibre composites by cylindrical punches with a conical tip. The aim is to develop an understanding of the penetration modes, and to contrast them with those of blunt punches. The outline of the study is as follows. We first report the experimental investigation of the penetration resistance of unidirectional and cross-ply composites along with optical microscopy and X-ray imaging to elucidate the penetration modes. Second, this understanding is used to develop a penetration model for these composites that includes the anisotropic plastic deformation of the composite and the appropriate fracture modes. Finally, the models are used to construct penetration mechanism maps which aid the design of optimal composite microstructures.

\section{Experimental protocol}

The aim of the experimental study was to investigate the resistance of both unidirectional (UD) and cross-ply UHMWPE composites against penetration by a cylindrical punch of radius $a$ and a conical tip characterised by half angle $\alpha$; see Fig. 1a.

\subsection{Composite manufacture and configurations}

Four grades of UHMWPE composites supplied by DSM under the tradename Dyneema ${ }^{\circledR}$ comprising SK76 UHMWPE fibres in a polyurethane matrix were employed in this investigation. The manufacturing steps employed by DSM for the cross-ply composites with a $\left[0^{\circ} / 90^{\circ}\right]$ stacking sequence of the plies were detailed in Russell et al. (2013) and Attwood et al. (2014), and here we summarise these steps in order to clarify the differences in the process used to manufacture the UD composites.

(i) A gel-spinning/hot drawing process (Smith et al., 1979; Smith and Lemstra, 1980) is used to produce highly orientated and highly crystalline fibres of diameter $17 \mu \mathrm{m}$.

(ii) Fibres are coated in a resin solution and are then formed into a $\left[0^{\circ} / 90^{\circ} / 0^{\circ} / 90^{\circ}\right]$ stack. A drying process removes the matrix solvent from the stack, and several stacks are placed on top of each other in order to form the desired laminate.

(iii) The laminate is hot-pressed with bonding of the layers achieved through partial melting of the matrix material. The fibre diameter is unchanged by the hot-pressing operation, although some fibres may change their cross-sectional shape. 
a

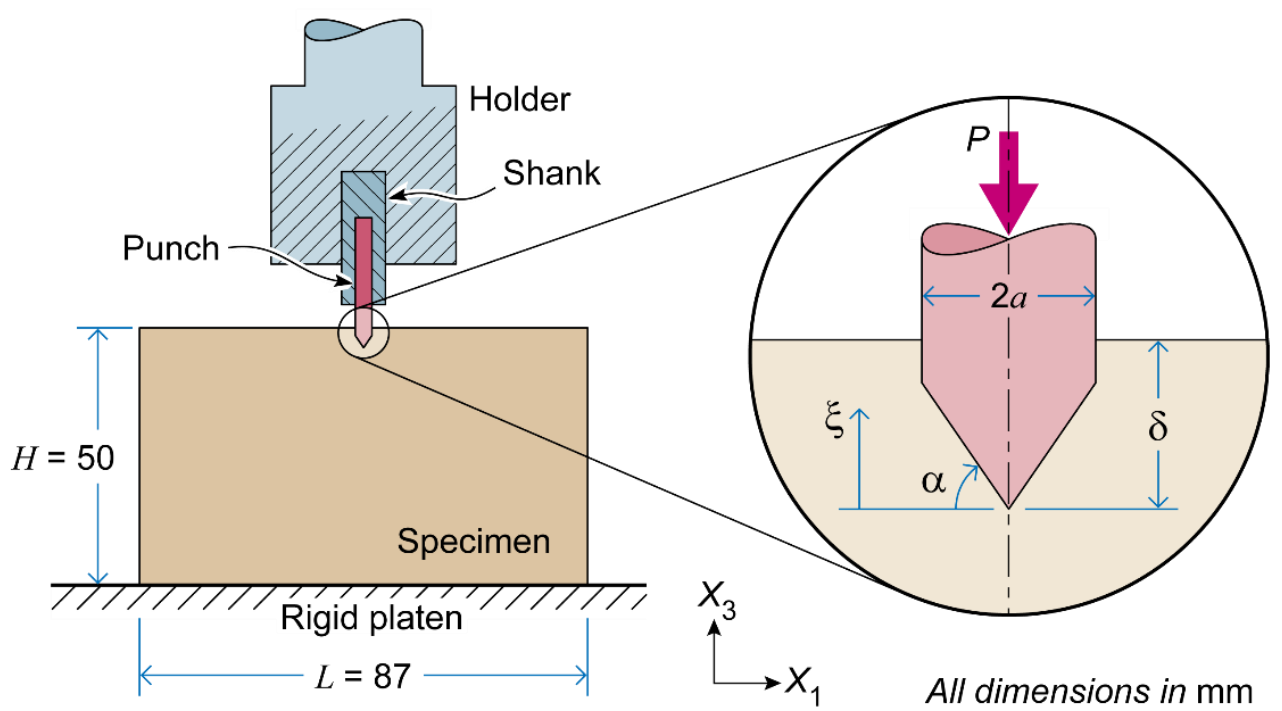

b
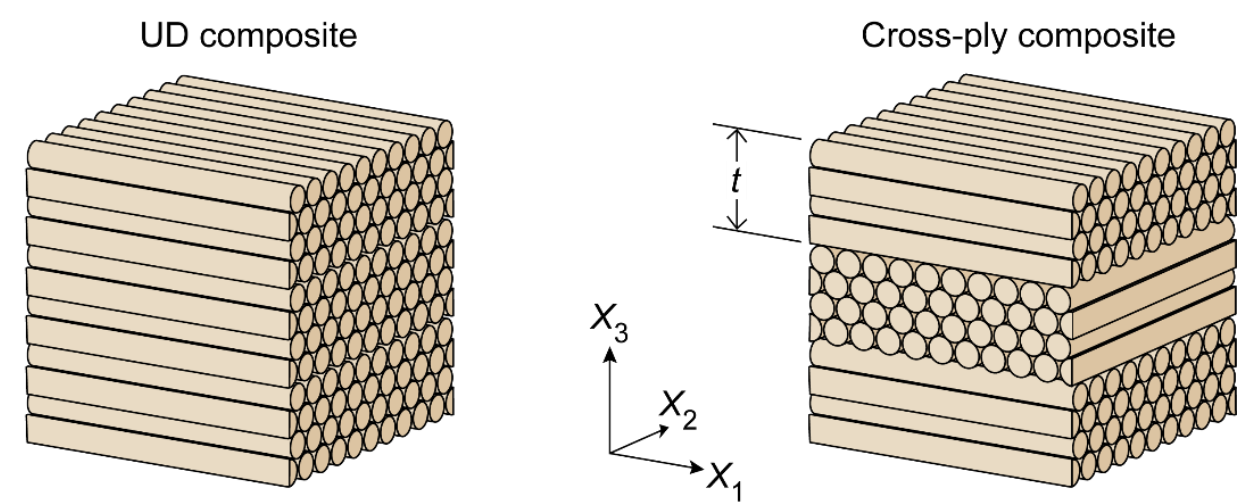

Figure 1: (a) Schematic of the penetration of UHMWPE composites by a sharp-tipped cylindrical punch. The inset shows details of the conical tip of the punch. (b) Sketches of the UD and cross-ply composites with the definition of the global Cartesian co-ordinate system $X_{i}$.

The DSM supplied all the cross-ply composites used in this study in plates of dimensions $400 \mathrm{~mm} \times 400 \mathrm{~mm}$ and $50 \mathrm{~mm}$ thick (i.e. the plies were stacked to give a $50 \mathrm{~mm}$ thick composite). Three grades of cross-ply composites were employed with ply thicknesses $t=$ $30 \mu \mathrm{m}, 60 \mu \mathrm{m}$ and $120 \mu \mathrm{m}$. The commercial designations of these composites are HB80, HB26 and HB25 respectively but throughout this study we refer to them by their ply thicknesses. With $X_{1}$ and $X_{2}$ denoting directions of a Cartesian co-ordinate system aligned with the orthogonal fibres of the cross-ply (Fig. 1b), dark field optical images of an $X_{1}-X_{3}$ plane through the three grades of the cross-ply composites are included in Fig. 2. The ply architecture and the closely packed fibres are clearly visible in these images.

An analogous hot-pressing process was used to manufacture the UD composites. Matriximpregnated UD plies were laid-up into a plate of size $100 \mathrm{~mm} \times 100 \mathrm{~mm}$ and $50 \mathrm{~mm}$ thick. These plies were then pressed together at a temperature of $127^{\circ} \mathrm{C}$ and a consolidation pressure of $21 \mathrm{MPa}$ for 20 minutes to produce a UD laminate. Again, we define a Cartesian co-ordinate system with $X_{1}$ aligned with the fibre direction and $X_{3}$ aligned with the transverse direction along which the composite was consolidated in the manufacturing process. All of the composite grades contained a fibre volume fraction $v_{f} \approx 83 \%$. The quasi-static tensile, shear and out-ofplane compression responses of the four grades of UHMWPE composites used in this study 
were reported by Attwood et al. (2014), and we shall use those property measurements to inform the material models in Section 4.2.



$x_{3}$<smiles>[Y]C1CCCC1</smiles>

Figure 2: Dark field optical micrographs of an $X_{1}-X_{3}$ plane through the three grades of the cross-ply Dyneema ${ }^{\circledR}$ composites with ply thicknesses (a) $t=30 \mu \mathrm{m}$, (b) $t=60 \mu \mathrm{m}$ and (c) $t=$ $120 \mu \mathrm{m}$.

\subsection{Measurements and imaging}

All measurements reported in the main body of this paper were conducted on cuboidal blocks of the UHMWPE composite of lateral dimension $L \times L=87 \mathrm{~mm} \times 87 \mathrm{~mm}$ and thickness $H=50 \mathrm{~mm}$ (Fig. 1a). These blocks were cut from the as-manufactured plates using a medium-fine blade band-saw and were sufficiently large that the measured penetration response was reasonably insensitive to further increases in the specimen size.

Penetration experiments were conducted as shown in Fig. 1a with both the UD and cross-ply composites penetrated in the $X_{3}$-direction by a cylindrical punch with a conical tip. The composite blocks were placed on a rigid backing and the penetration experiments were conducted in a screw-driven test machine with a holder as sketched in Fig. 1a to grip the punch. The penetration $\delta$ was defined as the depth of the tip below the surface of the composite block (Fig. 1a) and measured using a laser extensometer. The punch was displaced at a rate of $\dot{\delta}=$ $0.5 \mathrm{~mm} \mathrm{~min}^{-1}$ and the applied load $P$ was measured via the load cell of the test machine. The measurements are presented in terms of the applied nominal pressure $p \equiv P /\left(\pi a^{2}\right)$ and the normalized penetration $\bar{\delta} \equiv \delta / d$, where $d=17 \mu \mathrm{m}$ is the fibre diameter and a relevant length scale for both the UD and cross-ply composites. Measurements are reported for punches with diameters in the range $0.25 \mathrm{~mm} \leq 2 a \leq 4 \mathrm{~mm}$ with cone tip half angles $\alpha=30^{\circ}, 45^{\circ}$ and $60^{\circ}$. The punches for the UD composites were made from hardened silver steel ( 800 Vickers). However, the high pressure required to penetrate the cross-ply composites resulted in elastic buckling of the silver steel punches inside the cross-ply composites. Thus, TungstenCarbide/Cobalt (WC 94 Co $6 \mathrm{wt} \%$ ) rods with a modulus twice that of the silver steel punches were used for the penetration of the cross-ply composites. Nevertheless, buckling of the penetrator restricted the experiments on cross-ply composites to punches with diameters $2 a \geq$ $2 \mathrm{~mm}$. At-least 3 repeat tests were performed in each case to check the reproducibility of the measurements.

Both the UD and cross-ply composites penetration experiments were interrupted at regular intervals to conduct the optical and X-ray observations of the penetration modes. However, the protocol varied between the UD and cross-ply composites. The penetration mode of the UD composite involved splitting and separation of the fibres with no fibre breakage and only a small elastic deformation of the fibres. This implied that removal of the punch from the 
specimen resulted in negligible spring back and left the intact penetration cavity. This cavity was then filled with a solder powder ( $\mathrm{Sn} 60, \mathrm{~Pb} 38, \mathrm{Ag} 2 \mathrm{wt} \%$ ) with an average particle size of $30 \mu \mathrm{m}^{1}$. X-ray computed tomography (XCT) enabled three-dimensional (3D) reconstruction of the cavity around the punch. In addition, we also conducted optical imaging of interior $X_{1}-$ $X_{2}$ planes of the UD composites in order to visualise the fibre deformations around the indenter. This optical imaging involved peeling-off layers to expose the specimen interior and hence was a destructive process. Fibre layers of the specimen, with the penetration cavity filled with the solder powder, were peeled-off until a layer at a depth of approximately $26 a$ below the specimen surface was exposed. Then, another $1 \mathrm{~mm}$ or so of the specimen was abraded using a fine-grit (P800-P4000) in order to obtain a clean and smooth surface for imaging. The imaged area of this exposed specimen surface was then divided into a grid comprising approximately 200 squares. Each of these squares was imaged separately and the entire imaged area was then reconstructed by stitching together these sub-images. This procedure enabled us to obtain a relatively high resolution over a large area.

The cross-ply composites were penetrated by Tungsten-Carbide/Cobalt rods at significantly higher pressures. Elastic deformations of the fibres in these specimens meant that the inserted punches were tightly gripped within the specimen and their removal resulted in not only extensive damage of the specimen but also collapse of the penetration cavity. Thus, optical imaging of the specimen interior by peeling-off plies was not feasible. Moreover, since Tungsten-Carbide/Cobalt is nearly X-ray opaque, the shadow cast by the rod inhibited a 3D reconstruction of the specimen deformation around the punch. Instead, we used plain radiographs taken by imaging the specimen along the $X_{3}$-direction so as to infer some aspects of the penetration mode. Additional details of the X-ray imaging and analysis method are provided in Appendix A.

\section{Experiment measurements and observations}

We proceed to detail the measurements of the penetration response. The objectives are to characterize the effect of the punch radius $a$ and ply-thickness $t$ on the $p-\bar{\delta}$ response as well as to elucidate the penetration modes.

\subsection{Unidirectional composites}

The measured $p$ versus $\bar{\delta}$ responses for UD composites penetrated by punches of diameters in the range $0.25 \mathrm{~mm} \leq 2 a \leq 2 \mathrm{~mm}$ and a tip angle $\alpha=45^{\circ}$ are included in Fig. 3a. In each case two measured responses are included to illustrate the repeatability of the measurements. Two regimes are observed for all indenter diameters: regime $I$ where the pressure $p$ increases with penetration $\bar{\delta}$; and regime II where $p$ remains approximately constant and we denote this steady-state pressure by $p_{s s}$. The effect of the tip angle $\alpha$ is illustrated in Fig. $3 \mathrm{~b}$ for the $2 a=$ $2 \mathrm{~mm}$ punch. During the initial stage of penetration, decreasing $\alpha$ reduced the penetration pressure. However, the steady-state pressure $p_{s s}$ was unaffected by $\alpha$.

\footnotetext{
${ }^{1}$ The solder powder enhanced the X-ray contrast and improved the X-ray imaging of the penetrated UHMWPE composite specimen.
} 

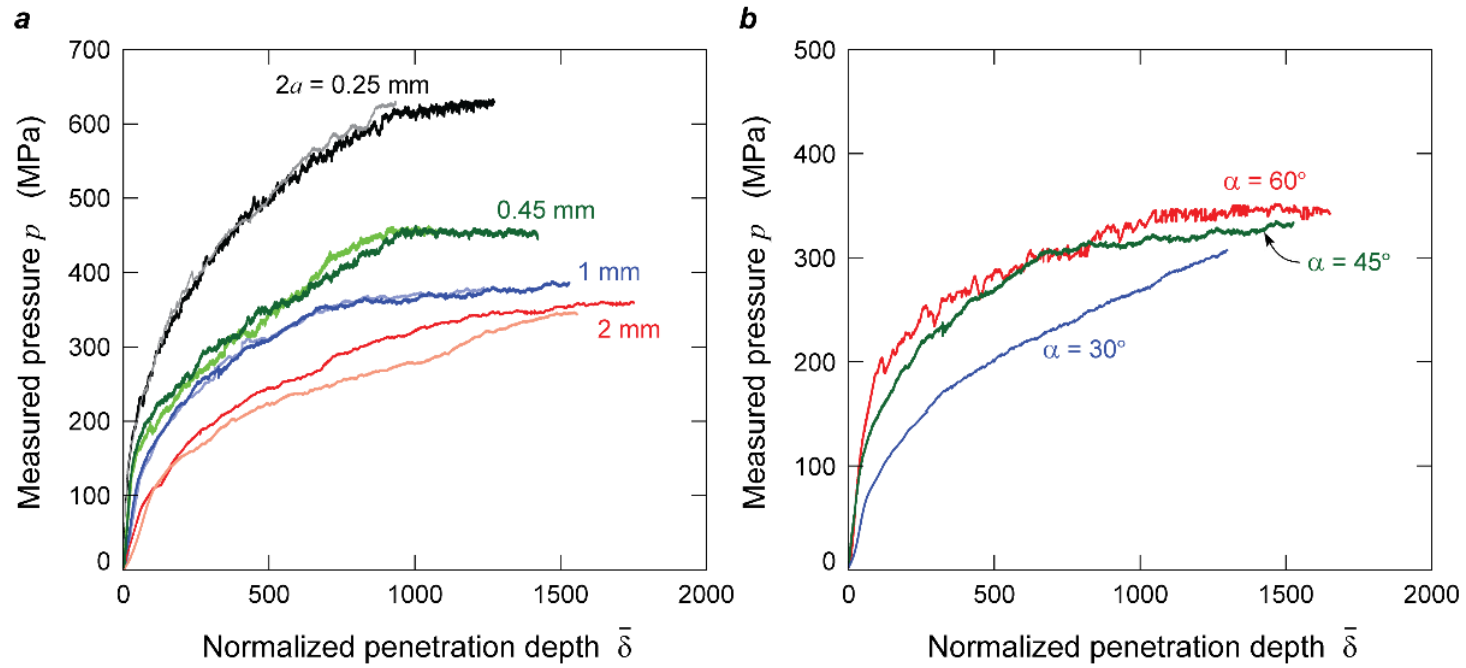

Figure 3: The measured pressure $p$ versus normalized penetration $\bar{\delta}$ responses for UD composites penetrated by (a) punches with diameters in the range $0.25 \mathrm{~mm} \leq 2 a \leq 2 \mathrm{~mm}$ with tip angle $\alpha=45^{\circ}$ and (b) a $2 a=2 \mathrm{~mm}$ diameter punch and three cone angles $\alpha$. In (a) two repeat measurements are included for each punch diameter.

The data in Fig. 3a indicates that the steady-state penetration pressure $p_{s s}$ increased with decreasing punch diameter. Such a penetration/indentation size effect is well-established for metals and related to micron-sized dislocation structures therefore only observed for punch/indenter diameters on the micron length scale. In the UD composite investigated here, the size effect is observed for millimetre size punch diameters even though the only relevant microstructural length scale is related to the $17 \mu \mathrm{m}$ diameter UHMWPE fibres. To investigate the source of this penetration size effect and understand the mode of penetration we include in Fig. 4 XCT images of a diametrical $X_{1}-X_{3}$ plane through the $2 a=2 \mathrm{~mm}$ punch $\left(\alpha=45^{\circ}\right)$ at three selected penetration depths into the specimen. The outline of the punch has been highlighted to clearly contrast the punch and the surrounding cavity within the UD composite. Cross-sectional images on $X_{1}-X_{2}$ planes at the marked heights are also included in each case to show the formation of a cavity in the form of a crack around the cylindrical punch. The length $2 \ell$ of this crack increases from the tip of the punch such that $\cot ^{-1}(\xi / \ell)>\alpha$, where $\xi$ is the distance measured upstream from the tip of the punch as shown in Fig. 1a: the crack length reaches a steady state value of $2 \ell_{s s}=9.50 \mathrm{~mm}$ for $\xi>3.2 \mathrm{~mm}$. We note that near the surface of the specimen there is a considerable pile-up of material. This pile-up is due to the formation of the cavity whose finite volume results in material being pushed up near the surface of the specimen. However, deep within the specimen, the increasing constraint results in negligible deformation in the $X_{3}$-direction with the formation of the cavity accommodated by in-plane deformation of the specimen.

In order to better understand the crack formation mechanism, Fig. 5 shows an optical micrograph of the $X_{1}-X_{2}$ plane at a depth $26 \mathrm{~mm}$ below the surface of the specimen penetrated by the $2 a=2 \mathrm{~mm}$ punch $\left(\alpha=45^{\circ}\right)$. This optical micrograph confirms that the crack seen in the XCT images of Fig. 4 is a mode-I crack along the fibre direction and a result of a splitting mode due to the wedging action of the penetrator, i.e. the penetrator induces tensile stresses $\Sigma_{22}$ that open a crack via matrix cracking with no associated fibre fracture. The material remains in contact with the surface of the cylindrical punch over a region defined by an included angle $2 \beta=68^{\circ}$ (Fig. 5), with the remaining cavity surface being traction-free. Thus, the wedging pressure generated over the region where the punch is in contact with the cavity provides the driving force for the extension of the crack. We emphasize here that friction 
between the cylindrical surface of the punch and the UHMWPE composite does not contribute significantly to the observed size effect. A simple work balance suggests that the penetration pressure with friction present is given by

$$
p_{s s}=p_{s s}^{0}+\frac{4 \tau_{f} \beta \delta}{\pi a}
$$

where $\tau_{f}$ is the frictional stress between the UHMWPE composite and the punch while $p_{S S}^{0}$ is the intrinsic steady-state penetration pressure. The frictional contribution (3.1) increases with increasing penetration depth and decreasing punch diameter. The data in Fig. 3 clearly shows that a steady-state is attained such that $p_{s s} \approx p_{s s}^{0}$ suggesting that $\tau_{f} \ll p_{s s}$. In fact, the measurements of the force to extract the punch from the specimen suggest that $\tau_{f}<2 \mathrm{MPa}$ confirming that friction between the punch and the composite plays a negligible role in these measurements. Moreover, the specimens employed in this study were sufficiently large so that no pull-in was observed at the specimen edges with the specimen size playing no role in the observed penetrator size effects. In fact, the analysis in Section 4 will show that the penetrator was accommodated within the specimen by elastic extension of the fibres with the observed penetration size effect for millimetre-sized diameter punches associated with the mode-I splitting toughness of the UD composite.
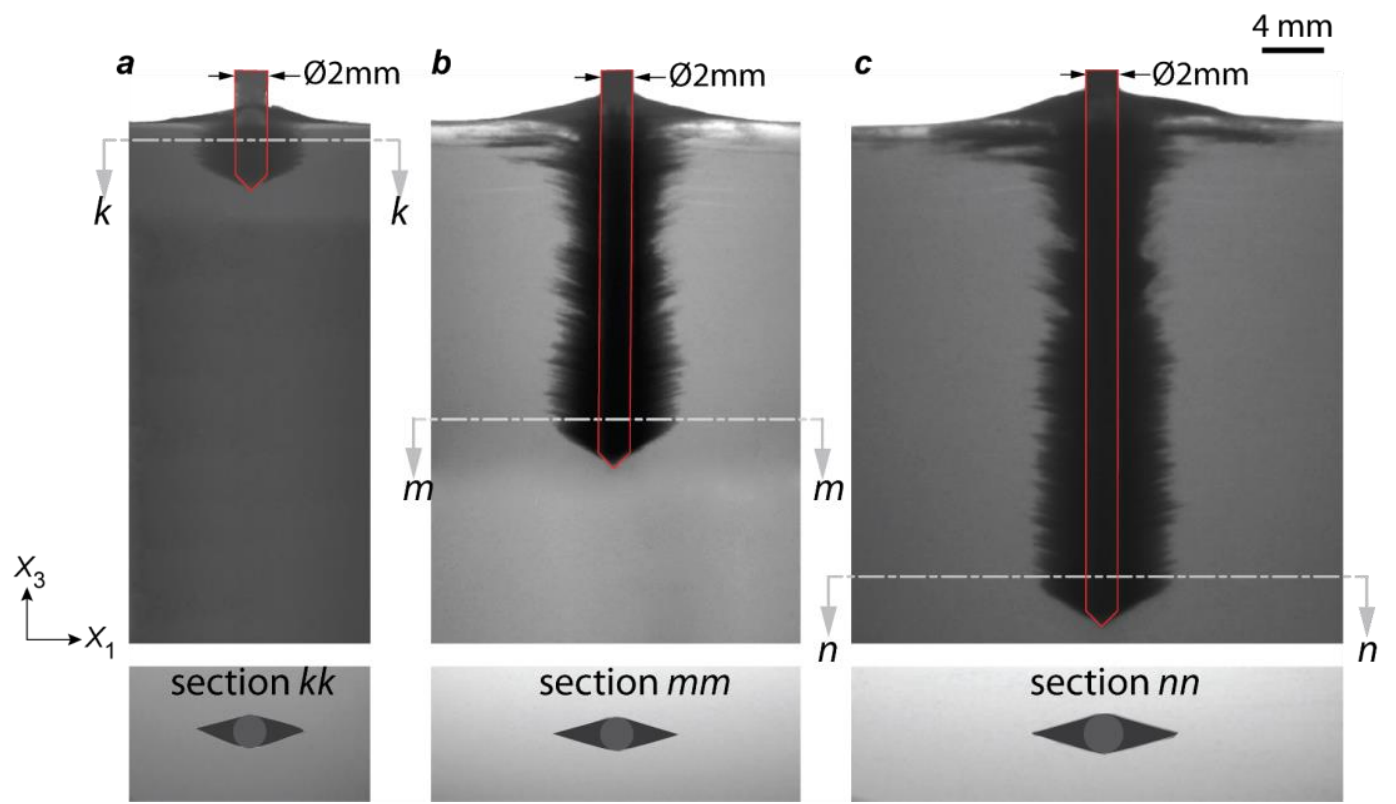

Figure 4: X-ray computed tomographic images of the UD composite penetrated by a $2 a=$ $2 \mathrm{~mm}$ diameter punch with cone angle $\alpha=45^{\circ}$. The images show a diametrical section through the punch for penetrations (a) $\delta=5 \mathrm{~mm}$, (b) $\delta=19 \mathrm{~mm}$ and (c) $\delta=27 \mathrm{~mm}$. In each case cross-sectional images of the $X_{1}-X_{2}$ plane at the marked heights are also included and the indenter is outlined in red for clarity. 


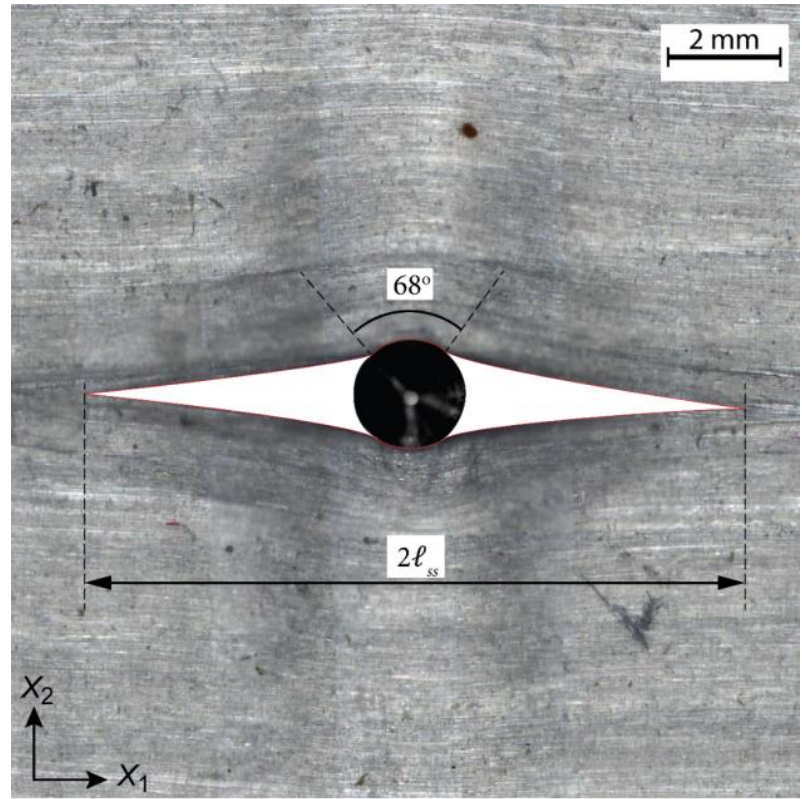

Figure 5: An optical micrograph of the $X_{1}-X_{2}$ plane at a depth $26 \mathrm{~mm}$ below the surface of the UD specimen penetrated by a $2 a=2 \mathrm{~mm}$ punch with cone angle $\alpha=45^{\circ}$. The mode-I crack length $2 \ell_{s s}=11.75 \mathrm{~mm}$ and the angle through which the composite conforms over the punch are labelled.

Experiments using different punch diameters indicated very similar deformation/fracture mechanisms to those observed above, and their micrographs are excluded for the sake of brevity. The key observations of the observed size effects are summarised Fig. 6a where the measured steady-state penetration pressure $p_{s s}$ and the associated normalized crack length $\ell_{s s} / a$ are plotted as a function of the punch diameter $2 a$. While the penetration pressure $p_{s s}$ increased with decreasing $a$, the opposite trend was observed for $\ell_{s s} / a$. Three repeat tests were conducted in each case and the error bars indicate the variation over these three measurements while the data points represent the mean measurement.
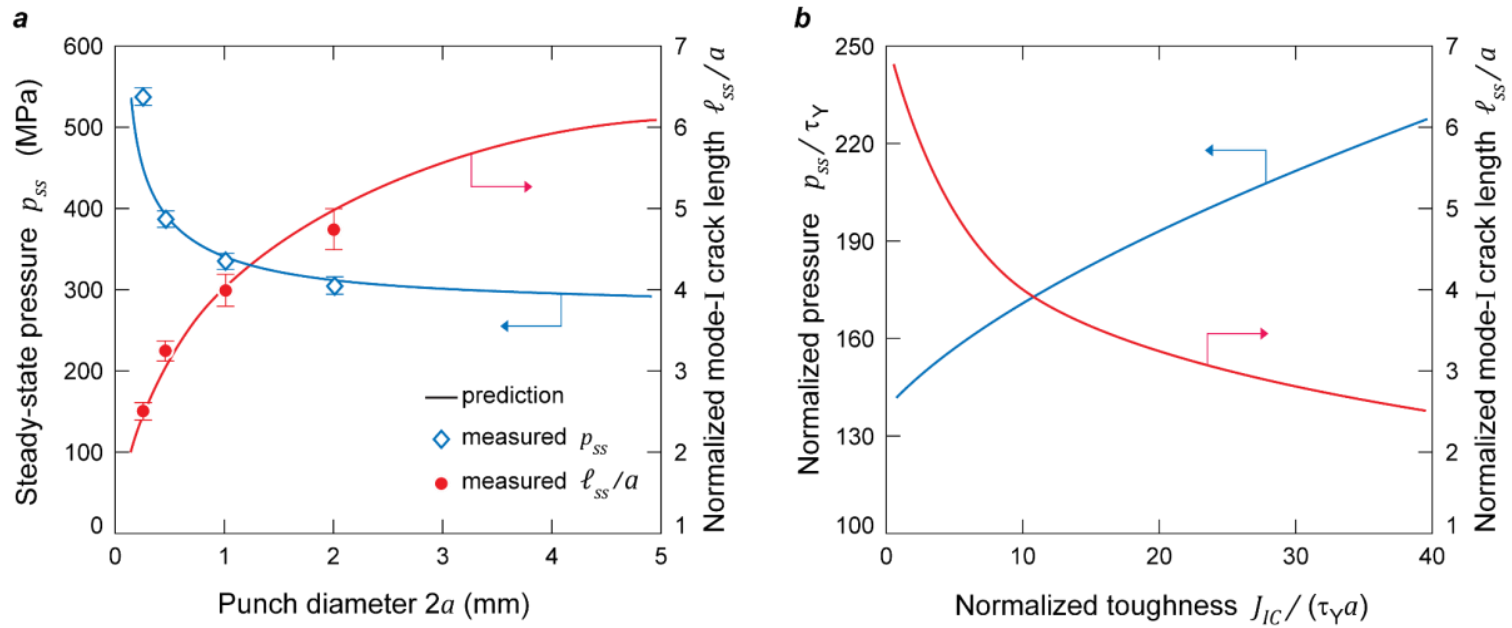

Figure 6: (a) Measurements and corresponding predictions of the steady-state penetration pressure $p_{s s}$ and normalized mode-I crack length $\ell_{s s} / a$ as a function of the punch diameter $2 a$ for the UD composite. The error bars indicate the variability between the 3 repeat measurements. (b) Predictions of the normalized penetration pressure $p_{s s} / \tau_{Y}$ and $\ell_{s s} / a$ as a function of the normalized toughness $J_{I C} /\left(\tau_{Y} a\right)$ for the UD composite. 


\subsection{Cross-ply composites}

The measured $p$ versus $\bar{\delta}$ response of the $t=60 \mu \mathrm{m}$ cross-ply composite is included in Fig. 7a for $\alpha=45^{\circ}$ punches of size in the range $2 \mathrm{~mm} \leq 2 a \leq 4 \mathrm{~mm}$. The responses are qualitatively similar to that of the UD composites except that the penetration pressures are significantly larger. A penetration size effect is again observed although weaker compared to the UD case. We emphasize that experiments on the cross-ply composites could only be performed for relatively large punch diameters with $2 a \geq 2 \mathrm{~mm}$, and it will be shown in Section 5 that this weak size effect is related to the large punch diameters. The ply thickness $t$, is an additional microstructural length scale in cross-ply composites. The effect of $t$ on the $p-\bar{\delta}$ response is illustrated in Fig. 7b where measurements are included for cross-ply composites with $t=$ $30 \mu \mathrm{m}, 60 \mu \mathrm{m}$ and $120 \mu \mathrm{m}$ penetrated by the $2 a=2 \mathrm{~mm}\left(\alpha=45^{\circ}\right)$ punch. A very strong size effect is now observed with the penetration pressure higher for composites with thinner plies over nearly the entire range of penetration depths.
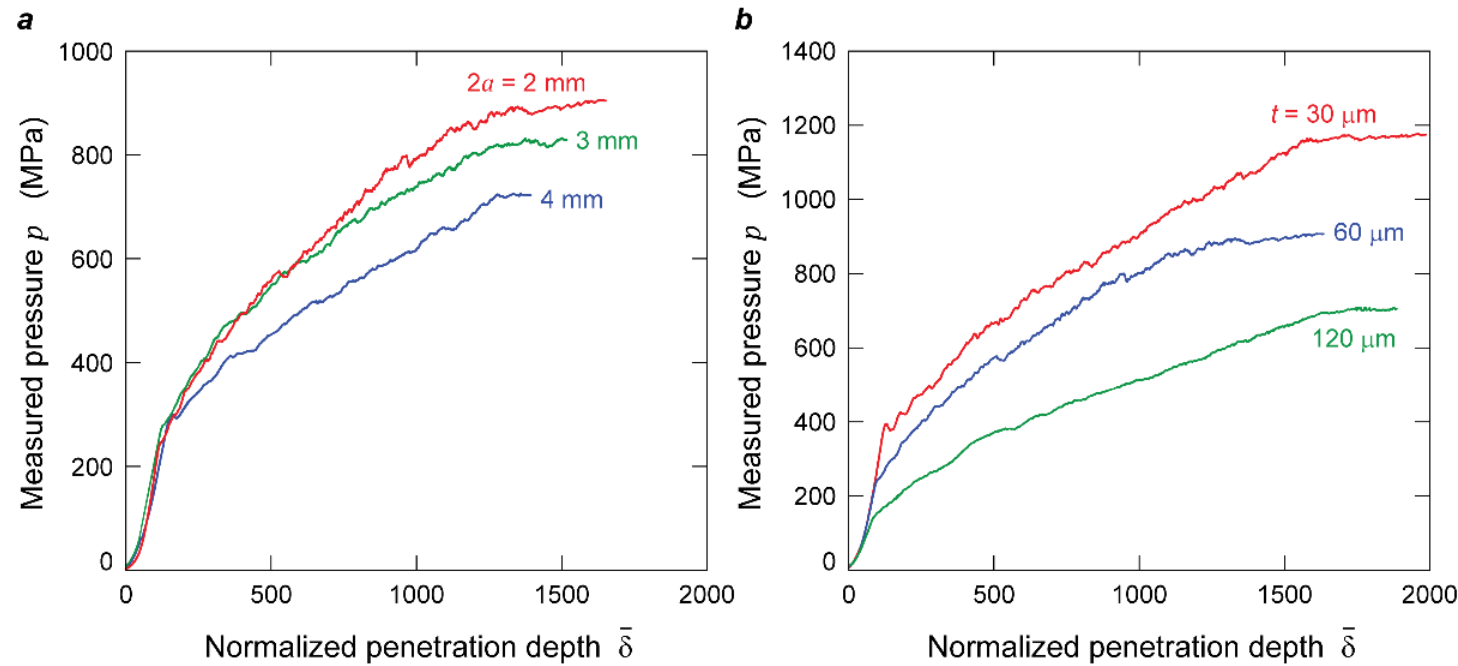

Figure 7: The measured pressure $p$ versus normalized penetration $\bar{\delta}$ responses for (a) the $t=$ $60 \mu \mathrm{m}$ cross-ply composite penetrated by punches with diameters in the range $2 \mathrm{~mm} \leq 2 a \leq$ $4 \mathrm{~mm}$ and (b) cross-ply composites with ply thicknesses $t=30 \mu \mathrm{m}, 60 \mu \mathrm{m}$ and $120 \mu \mathrm{m}$ penetrated by the $2 a=2 \mathrm{~mm}$ punch. In all cases the cone angle of the punch tip was $\alpha=45^{\circ}$.

XCT imaging of the cross-ply composites penetrated by the Tungsten-Carbide/Cobalt penetrators was not feasible as discussed earlier. Thus, visualisation of the deformation/fracture modes are restricted to plain radiographs such as the one shown in Fig. 8 for the $t=60 \mu \mathrm{m}$ cross-ply composite penetrated by the $2 a=3 \mathrm{~mm}\left(\alpha=45^{\circ}\right)$ punch. In this image we observe a central circular dark zone with an outer concentric circular partially illuminated region. These are the umbra and penumbra shadows of the penetrator that is opaque to the X-rays. In addition, two orthogonal cracks are seen to emanate from the central zone each akin to the single crack seen for the UD composite. We argue that these cracks are mode-I cracks similar to the UD composite but now in the alternating $\left[0^{\circ} / 90^{\circ}\right]$ plies. A quantitative interpretation of this image to extract the crack length is complex and readers are referred to Appendix A for details. The analysis indicates that the central dark zone is an enlarged image of the circular cross-section of the punch and obscures some part of the orthogonal mode-I cracks. This gives an optical illusion that the cavity formed around the punch conforms over nearly the entire cylindrical surface of the punch. Note that the scale on Fig. 8 is for the image on the detector plane and cannot be directly used to infer $\ell_{s s}$ : rather a more involved analysis as detailed in Appendix A needs to be conducted from which we infer that the mode-I crack length $2 \ell_{s s}=14 \mathrm{~mm}$. 
Measurements of the steady-state pressure $p_{s S}$ and associated normalized crack lengths $\ell_{s s} / a$ (estimated using the procedure described in Appendix A) are summarised in Fig. 9a as a function of $2 a$ for the $t=60 \mu \mathrm{m}$ cross-ply composite penetrated by $\alpha=45^{\circ}$ punches. The results are qualitatively similar to the UD composite with $p_{s s}$ increasing with decreasing $2 a$ while $\ell_{s s} / a$ displays the opposite trend. The corresponding $p_{s s}$ and $\ell_{s s} / a$ measurements for cross-ply composites penetrated by the $2 a=2 \mathrm{~mm}$ punch $\left(\alpha=45^{\circ}\right)$ are included in Fig. $9 \mathrm{~b}$ as a function of the ply thickness $t$. The trends are similar to the dependence on the punch diameter with $p_{s s}$ increasing with decreasing $t$ while $\ell_{s s} / a$ displays the opposite trend although the dependence of the mode-I crack length on the ply thickness is relatively mild.

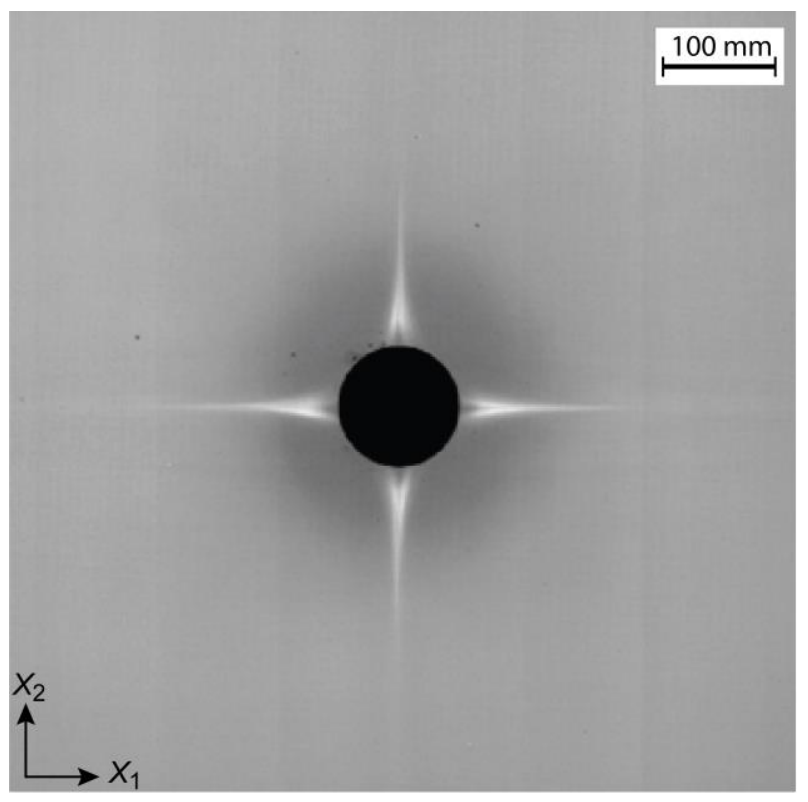

Figure 8: A plain radiograph of an $X_{1}-X_{2}$ plane of the $t=60 \mu \mathrm{m}$ cross-ply composite penetrated by the $2 a=3 \mathrm{~mm}$ punch. The scale bar represents the size of the image on the detector plane; see Appendix A for further details.
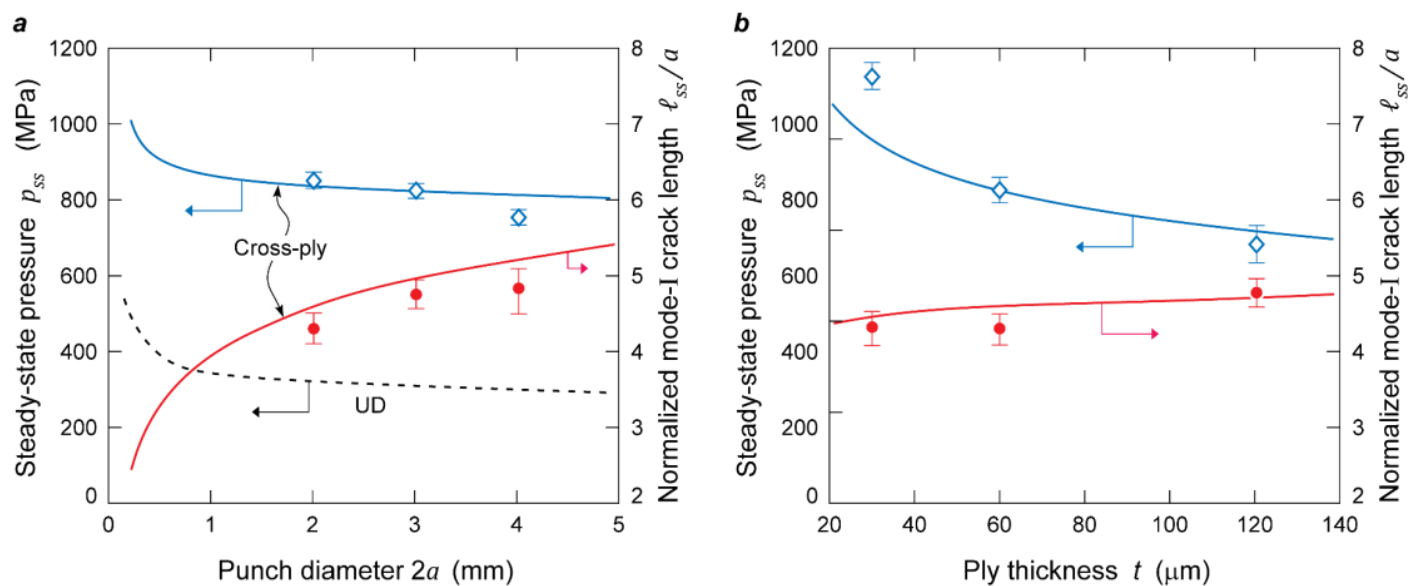

Figure 9: Measurements and corresponding predictions of the steady-state penetration pressure $p_{s s}$ and normalized mode-I crack length $\ell_{s s} / a$ as a function of (a) the punch diameter $2 a$ for the $t=60 \mu \mathrm{m}$ cross-ply composite and (b) the ply thickness $t$ for penetration by the $2 a=2 \mathrm{~mm}$ punch. In all cases the cone angle $\alpha=45^{\circ}$ and the error bars indicate the variability between the 3 repeated measurements. 


\section{Analysis of the steady-state penetration of the unidirectional composite}

Our aim here is to develop an understanding of the key physical phenomena that govern the penetration response including the observed size effect of the diameter of the punch. With this in mind, we develop a semi-analytical model for the steady-state penetration motivated by the experimental observations reported above. In order to motivate this analysis, we summarise the observed deformation and fracture modes for the penetration of UD and cross-ply composites by a sharp-tipped punch in Figs. 10a and 10b, respectively. The UD composites form a single mode-I crack with the punch wedging open a crack along the fibres, i.e. mode-I splitting by matrix cracking with no fibre fracture. The cross-ply composites also deform in a similar mode with orthogonal mode-I splitting cracks forming in the alternating $\left[0^{\circ} / 90^{\circ}\right]$ plies. These cracks however cause a mismatch in deformation due to crack opening in the $X_{2}$ and $X_{1}$ directions in the $0^{\circ}$ and $90^{\circ}$ plies, respectively. The high modulus of the fibres prevents large localised fibre extensions and thus these deformations cannot be accommodated by ply deformations. Rather we anticipate delamination to occur between each pair of adjacent $0^{\circ}$ and $90^{\circ}$ plies to accommodate this incompatible deformation. We note that the inability to acquire XCT images for the cross-ply composites meant this delamination could not be directly visualised. Moreover, deep within the specimen the delamination occurs via mixed mode-II/III fracture with no separation between the plies in the $X_{3}$ - direction. Thus, these delamination cracks are unlikely to be visualised via X-ray tomography. In Fig. 10b, we depict this delamination to be a circular zone of radius $b_{S S}$. We emphasize that in both UD and cross-ply composites no fibre fracture was observed for penetration by a sharp-tipped punch.

By contrast, the penetration of the composites by a blunt punch occurs by radically different mechanisms as shown in Figs. 10c and 10d for UD and cross-ply composites, respectively; see Attwood et al. (2016) and O'Masta et al. (2016) for further details. Briefly, the UD composites deform in a plastic indentation mode involving matrix shear with minimal fibre deformation. By contrast, the compressive indentation stresses induced in the cross-ply composite by a blunt punch generates a state of indirect tension (Attwood et al., 2014), i.e. the tensile stresses are generated in the fibre directions in the alternating $\left[0^{\circ} / 90^{\circ}\right]$ plies. Continued penetration of the cross-ply composite then occurs by fibre fracture and the elastic recoil (and thus removal) of the failed plies; readers are referred to Attwood et al. (2016) and O'Masta et al. (2016) for further details. This blunt punch penetration mode results in a penetration resistance that is independent of both the punch size and ply thickness (Attwood et al., 2016).

Using the above insight into the deformation and fracture processes for penetration by a sharptipped punch, we proceed in this section to develop a semi-analytical model for the steady-state penetration of UD composites and extend it to cross-ply composites in Section 5. 
a


C

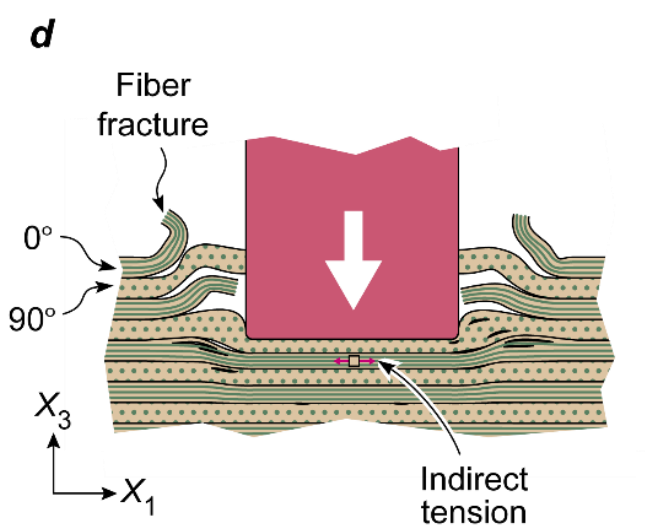

Figure 10: Sketches of the penetration modes of the (a) UD and (b) cross-ply composites by a sharp-tipped punch. The mode-I cracks along the fibres and the delamination zones in the crossply composite are labelled. Sketches of the deformation/failure modes for indentation of (c) UD and (d) cross-ply composites by a blunt punch.

\subsection{Penetration analysis}

Consider a rigid cylindrical punch of radius $a$ with a conical tip pushed into a semi-infinite block of the UD composite, as shown in Fig. 1a. In line with the measurements and discussion in Section 3 we shall neglect the role of friction between the punch and the composite and thus model the punch as frictionless. The composite splits open creating a mode-I crack along the fibres at the tip of the punch. A detailed solution for the deformation and fracture at the punch 
tip requires a full 3D calculation of considerable complexity. Here, in keeping with our aim of understanding the key physical phenomena, we propose a simplified analysis for the steadystate penetration pressure $p_{s s}$ of the fibre composite. The analysis is motivated by the model of Shergold and Fleck (2004) for the penetration of soft elastomers. The steady-state advance of the punch by an increment $d \delta$ involves the transformation of a slice of thickness $d \delta$ of the material from its undeformed upstream state to a cracked downstream state with a plane strain crack of length $2 \ell_{s s}$ that is opened by the wedging action of the punch of radius $a$ (Fig. 10a). The energy required to transform a slab of thickness $d \delta$ from its upstream to downstream state is provided by the work done in advancing the punch and an energy balance dictates

$$
p_{s S}\left(\pi a^{2}\right) d \delta=2 d \delta \int_{0}^{\ell} J_{I} d \ell+U_{D} d \delta .
$$

Here $J_{I}$ is the mode-I inter-fibre splitting toughness while $U_{D}$ is the energy required to open the crack of length $2 \ell$ to accommodate a cylinder of radius $a$ (Fig. 10a) in a unit thickness slab of the composite. At steady-state penetration, the crack just arrests upon reaching a length $2 \ell_{s s}$ and is thus at a state of incipient advance. Recalling the energy release rate definition of the deformation $J$ integral (Anderson, 2005) it follows that

$$
J_{I}=-\frac{1}{2} \frac{\partial U_{D}}{\partial \ell}
$$

For a given UHMWPE composite with a shear strength $\tau_{Y}$, it is convenient to express $U_{D}$ as

$$
U_{D} \equiv \tau_{Y} a^{2} \mathcal{F}\left(\frac{\ell}{a}, \mathcal{M}\right)
$$

where the non-dimensional function $\mathcal{F}(\ell / a, \mathcal{M})$ needs to be numerically evaluated for the given composite material properties represented here by $\mathcal{M}$. Assuming that the mode-I toughness is independent of crack advance (i.e. no R-curve effect) and denoting this constant toughness as $J_{I C}$, the steady-state penetration pressure follows from (4.1) as

$$
p_{s S}=\frac{2}{\pi}\left(\frac{J_{I C}}{a}\right)\left(\frac{\ell_{s S}}{a}\right)+\frac{\tau_{Y}}{\pi} \mathcal{F}\left(\frac{\ell_{S S}}{a}, \mathcal{M}\right),
$$

with (4.2) specifying that the steady-state crack length $\ell_{s S}$ satisfies the relation

$$
J_{I C}=-\left.\frac{\tau_{Y} a}{2} \frac{\partial \mathcal{F}}{\partial(\ell / a)}\right|_{\ell=\ell_{s S}} .
$$

It now remains to specify the procedure for the calculation of $U_{D}$ (or equivalently $\mathcal{F}$ ). We do this in two steps: first we detail a constitutive model for unidirectional UHMWPE composites and then discuss the numerical procedure to evaluate $U_{D}$ using this constitutive model.

\subsection{Constitutive model for the UD composite}

The deformation of the UHMWPE composite around the punch involves large shear strains and fibre rotations; see Fig. 5. The deformation of the SK76 UHMWPE fibres is purely elastic and thus shearing of the matrix is restricted along planes dictated by the fibre directions. For example, consider the deformation of the UD composite by simple shear via the application of a shear strain $\gamma_{13}$ as shown in Fig. 11a. The composite deforms primarily by the relative displacement of the fibres in the $X_{1}$-direction by the shear straining of the intermediate matrix. As a consequence, while the material is subjected to a rotation $\gamma_{13} / 2$ the fibres undergo no rotation. This is akin to crystals that deform by shearing along slip planes due to the motion of dislocations and thus we proceed to develop a constitutive model for unidirectional UHMWPE composites motivated by crystal plasticity (Asaro, 1983; Hill and Rice, 1972). This crystal plasticity formulation for UD composites is expected to accurately account for the large plastic shear strains and associated fibre rotations in these composites. Other equivalent 
approaches such as the rebar approach proposed by Nazarian and Zok (2014) are also feasible although the crystal plasticity approach is ideally suited to model the pressure dependency of plastic flow in UHMWPE composites as discussed subsequently.

\subsubsection{Kinematics}

Following the usual notions in crystal plasticity, we define a lattice labelled by the fibre direction. The material is presumed to flow through the lattice due to shearing of the matrix and then the lattice with the embedded material undergoes elastic deformations and rigid body rotations. Using Cartesian tensor notation, the material deformation gradient $F_{i j}$ is written as a multiplicative decomposition of the elastic and plastic deformations characterised by $F_{i j}^{*}$ and $F_{i j}^{p}$, respectively such that

$$
F_{i j}=F_{i k}^{*} F_{k j}^{p}
$$

Thus, there exists an intermediate configuration arising from the pure plastic deformation $F_{i j}^{p}$ of the material. The deformations embodied in $F_{i j}^{p}$ are described in terms of shearing along crystallographic slip systems set by the fibre direction. A particular slip system $(\beta)$ is specified by vectors $\left(s_{i}^{(\beta)}, m_{i}^{(\beta)}\right)$ where $s_{i}^{(\beta)}$ specifies the slip direction and $m_{i}^{(\beta)}$ is the slip plane normal in the undeformed configuration. These vectors convect with the lattice so that in the deformed lattice they become

$$
s_{i}^{*(\beta)}=F_{i j}^{*} s_{j}^{(\beta)} \quad \text { and } \quad m_{i}^{*(\beta)}=m_{j}^{(\beta)}\left(F_{j i}^{*}\right)^{-1} .
$$

With the fibres aligned with the $X_{1}$-direction in the undeformed configuration, the $N=6$ slip systems in the UD composite are listed in Table 1 and selected systems are illustrated in Fig. $11 \mathrm{~b}$. The slip systems $\beta=1$ to 4 involve shearing in the $X_{i}$ directions while the $\beta=$ 5 and 6 systems involve shearing on planes oriented at $\pm 45^{\circ}$ with respect to $X_{2}$ direction with no shear component along the $X_{1}$ (fibre) direction. These slip systems accommodate all possible modes of deformation that do not involve fibre deformation. The plastic component of the deformation gradient associated with shearing on the slip systems then follows as

$$
\dot{F}_{i k}^{p}\left(F_{k j}^{p}\right)^{-1}=\sum_{\beta=1}^{N} \dot{\gamma}^{(\beta)} s_{i}^{(\beta)} m_{j}^{(\beta)},
$$

where $\dot{\gamma}^{(\beta)}$ is the shear rate on slip system $(\beta)$.

Table 1: The six slip systems in the UD composites as specified by $\left(s_{i}^{(\beta)}, m_{i}^{(\beta)}\right)$. The unit vectors are denoted as $(a, b, c)$ where $a, b, c$ are components in the $X_{1}, X_{2}$ and $X_{3}$-directions, respectively.

\begin{tabular}{|l|l|l|l|}
\hline \multicolumn{2}{|l|}{ Slip direction } & \multicolumn{3}{l|}{ Slip plane normal } \\
\hline$S^{(1)}$ & $(1,0,0)$ & $m^{(1)}$ & $(0,1,0)$ \\
\hline$S^{(2)}$ & $(1,0,0)$ & $m^{(2)}$ & $(0,0,1)$ \\
\hline$s^{(3)}$ & $(0,1,0)$ & $m^{(3)}$ & $(0,0,1)$ \\
\hline$S^{(4)}$ & $(0,0,1)$ & $m^{(4)}$ & $(0,1,0)$ \\
\hline$S^{(5)}$ & $(0,1 / \sqrt{2}, 1 / \sqrt{2})$ & $m^{(5)}$ & $(0,-1 / \sqrt{2}, 1 / \sqrt{2})$ \\
\hline$S^{(6)}$ & $(0,-1 / \sqrt{2}, 1 / \sqrt{2})$ & $m^{(6)}$ & $(0,-1 / \sqrt{2},-1 / \sqrt{2})$ \\
\hline
\end{tabular}


a
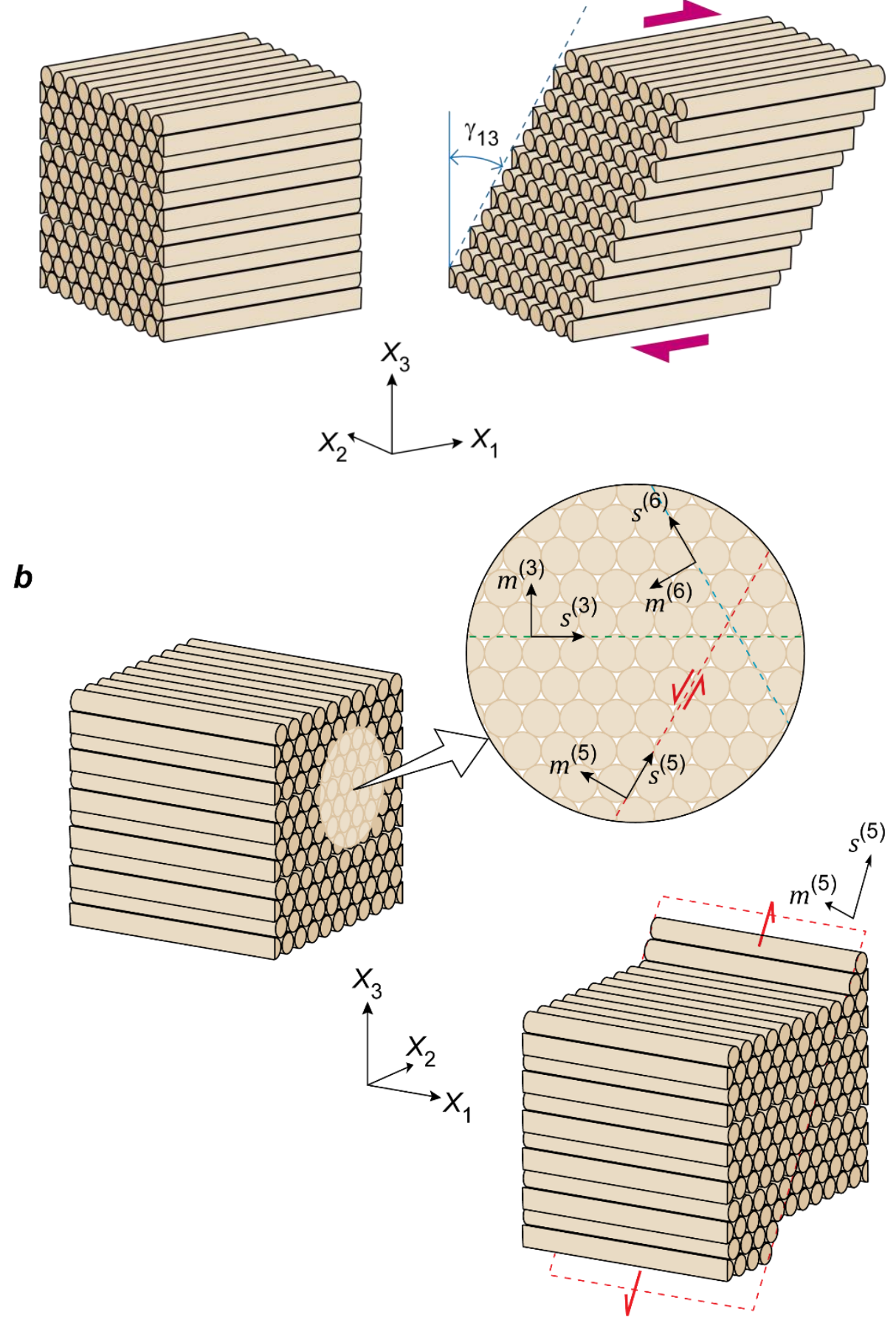

Figure 11: (a) The UD composite subjected to a simple shear $\gamma_{13}$ resulting in no rotation of the fibres. (b) A sketch of the UD composite showing some selected slip systems with their associated directions $\left(s_{i}^{(\beta)}, m_{i}^{(\beta)}\right)$ labelled.

\subsubsection{Elastic-plastic relations}

The experiments of Attwood et al. (2014) suggested that the deformation of UHMWPE composites is adequately characterised by an anisotropic elastic and pressure dependent plastic relation. Here we propose elastic-plastic constitutive relations within the crystal plasticity context motivated by the experimental findings of Attwood et al. (2014). 
Let $\sigma_{i j}$ denote the Cauchy stress and we introduce a material stress measure $\Sigma_{i j}$ for the intermediate configuration such that

$$
\Sigma_{i j}=\operatorname{det}\left(F_{i j}^{*}\right)\left(F_{i k}^{*}\right)^{-1} \sigma_{k l}\left(F_{j l}^{*}\right)^{-1} .
$$

This stress measure $\Sigma_{i j}$ is work conjugate to an elastic Green-Lagrange strain defined as

$$
E_{i j}^{*}=\frac{1}{2}\left(F_{k i}^{*} F_{k j}^{*}-\delta_{i j}\right)
$$

where $\delta_{i j}$ is the Kronecker delta. Following Attwood et al. (2014) we specify a linear elastic constitutive relation of the form $E_{i j}^{*}=C_{i j k l} \Sigma_{k l}$ in terms of the compliance tensor $C_{i j k l}$. Further, the UD composite is assumed to be transversely isotropic with the $X_{1}$ fibre direction normal to the plane of isotropy. The elastic law is then written in the form

$$
\left(\begin{array}{l}
E_{11}^{*} \\
E_{22}^{*} \\
E_{33}^{*} \\
E_{23}^{*} \\
E_{13}^{*} \\
E_{12}^{*}
\end{array}\right)=\left(\begin{array}{cccccc}
\frac{1}{E_{f}} & -\frac{v_{12}}{E_{f}} & -\frac{v_{12}}{E_{f}} & 0 & 0 & 0 \\
-\frac{v_{12}}{E_{f}} & \frac{1}{E_{m}} & -\frac{v_{23}}{E_{m}} & 0 & 0 & 0 \\
-\frac{v_{12}}{E_{f}} & -\frac{v_{23}}{E_{m}} & \frac{1}{E_{m}} & 0 & 0 & 0 \\
0 & 0 & 0 & \frac{1+v_{23}}{E_{m}} & 0 & 0 \\
0 & 0 & 0 & 0 & \frac{1}{2 G_{12}} & 0 \\
0 & 0 & 0 & 0 & 0 & \frac{1}{2 G_{12}}
\end{array}\right)\left(\begin{array}{c}
\Sigma_{11} \\
\Sigma_{22} \\
\Sigma_{33} \\
\Sigma_{23} \\
\Sigma_{13} \\
\Sigma_{12}
\end{array}\right) .
$$

Here $E_{f}$ and $E_{m}$ are the Young's moduli in the fibre and transverse directions, respectively, while $v_{12}$ and $v_{23}$ are the Poisson's ratios in the $X_{1}-X_{2}$ and $X_{2}-X_{3}$ planes, respectively. The shear modulus in the $X_{1}-X_{2}$ plane is denoted as $G_{12}$.

It now remains to specify the constitutive relations for the plastic slip rate $\dot{\gamma}^{(\beta)}$. Significant numerical difficulties are associated with determining the active slip systems and the amount of slip on each of these systems if a rate independent model is employed for $\dot{\gamma}^{(\beta)}$. Thus, Asaro and Needleman (1985) proposed a simple rate dependent crystal plasticity formulation that provides a good approximation to the rate independent limit and we employ their methodology here. Plastic deformation due to shearing on each slip system $(\beta)$ depends on the resolved shear stress

$$
\tau^{(\beta)}=s_{i}^{*(\beta)} \sigma_{i j} m_{j}^{*(\beta)} .
$$

However, unlike metallic crystals, plastic flow in the UHMWPE composites is pressure dependent as shown by the experiments of Attwood et al. (2014) and Chocron et al. (2014). Attwood et al. (2014) demonstrated that this pressure dependency is adequately characterised by a friction coefficient and based on these findings we define an effective resolved shear stress as

$$
\hat{\tau}^{(\beta)}= \begin{cases}\max \left[\left(\left|\tau^{(\beta)}\right|-\mu p\right), 0\right] & p \geq 0 \\ \left|\tau^{(\beta)}\right| & p<0 .\end{cases}
$$


The pressure dependency is primarily associated with deformations of the matrix and hence the pressure $p$ is a measure of the pressure absent the fibre stresses. This pressure is thus defined in terms of the material stresses in the intermediate configuration as

$$
p \equiv-\frac{1}{2}\left(\Sigma_{22}+\Sigma_{33}\right)
$$

and the non-negative friction parameter $\mu$ quantifies the pressure sensitivity of the composite. Then following Asaro and Needleman (1985), the shear rate $\dot{\gamma}^{(\beta)}$ is specified by a rate dependent law as

$$
\dot{\gamma}^{(\beta)}=\dot{\gamma}_{0}\left(\frac{\hat{\tau}^{(\beta)}}{\tau_{Y}}\right)^{1 / m} \operatorname{sgn}\left[\tau^{(\beta)}\right],
$$

where $\tau_{Y}$ is the shear strength with the pressure $p=0, \dot{\gamma}_{0}$ is a reference strain rate and $m$ is a rate sensitivity exponent such that the rate independent limit is retrieved for $m \rightarrow 0$. The deformation energy rate per unit volume of the undeformed composite can then be calculated as $\dot{\mathcal{E}}=\mathrm{S}_{i j} \dot{E}_{i j}$ where the total Green-Lagrange strain is defined as

$$
E_{i j}=\frac{1}{2}\left(F_{k i} F_{k j}-\delta_{i j}\right)
$$

and its work-conjugate $2^{\text {nd }}$ Piola-Kirchhoff stress is given by

$$
\mathrm{S}_{i j}=\operatorname{det}\left(F_{i j}^{*}\right)\left(F_{i k}\right)^{-1} \sigma_{k l}\left(F_{j l}\right)^{-1} .
$$

This completes the specification of the crystal plasticity constitutive description of the UD composite.

\subsubsection{Material properties}

Unless otherwise specified, all calculations used parameters for the Dyneema ${ }^{\circledR}$ composites described in Section 2.1 These material parameters are based on measurements reported in Russell et al. (2013) and Attwood et al. (2014). The moduli of the composite in the fibre and transverse directions are $E_{f}=80 \mathrm{GPa}$ and $E_{m}=10 \mathrm{GPa}$, respectively while the Poisson's ratios are $v_{12}=0$ and $v_{23}=0.5$ with $G_{12}=3 \mathrm{GPa}$. The shear strength of the composites with a polyurethane matrix is $\tau_{Y}=2 \mathrm{MPa}$ while the pressure sensitivity co-efficient is $\mu=0.05$. The rate sensitivity parameters are chosen to approximately recover the rate independent limit and thus we take $\dot{\gamma}_{0}=0.1 \mathrm{~s}^{-1}$ and $m=0.1$. There are no reported measurements of the toughness $J_{I C}$ of UHMWPE composites and this parameter shall be selected in order to obtain agreement with the penetration measurements reported here. In addition, parametric studies for the dependence of the predictions on $J_{I C}$ are also reported.

\subsection{Finite element calculation of the deformation energy}

We wish to calculate the energy $U_{D}$ required to open a crack of length $2 \ell$ to accommodate a cylinder of radius $a$ (Fig. 10a) in a unit thickness slab of the composite. This is required to calculate the steady-state penetration pressure $p_{s S}$ and, as discussed in Section 4.1, this occurs with negligible deformation in the $X_{3}$-direction. Thus, it suffices to consider the plane strain problem sketched in Fig. 12a with deformation of the slab constrained to occur in the $X_{1}-X_{2}$ plane and the crack lying along the $X_{1}$-direction. Using symmetry considerations, we only model one quadrant of the slab with the centre of the punch located at the midpoint of the crack. For the elastic-plastic composite constitutive model described above, the deformation energy $U_{D}$ is in general dependent on the deformation path pursued to insert the cylindrical punch within the crack. The penetration of the conical tipped punch occurs by the opening and growth of the crack driven by the expansion of a cylindrical insert from an initial radius $r=0$ at the 
conical tip to the radius $r=a$ of the punch. Here we try to emulate this deformation path as closely as possible by calculating $U_{D}$ with loading specified by the expansion of a cylinder located at the centre of the $\mathrm{crack}^{2}$.
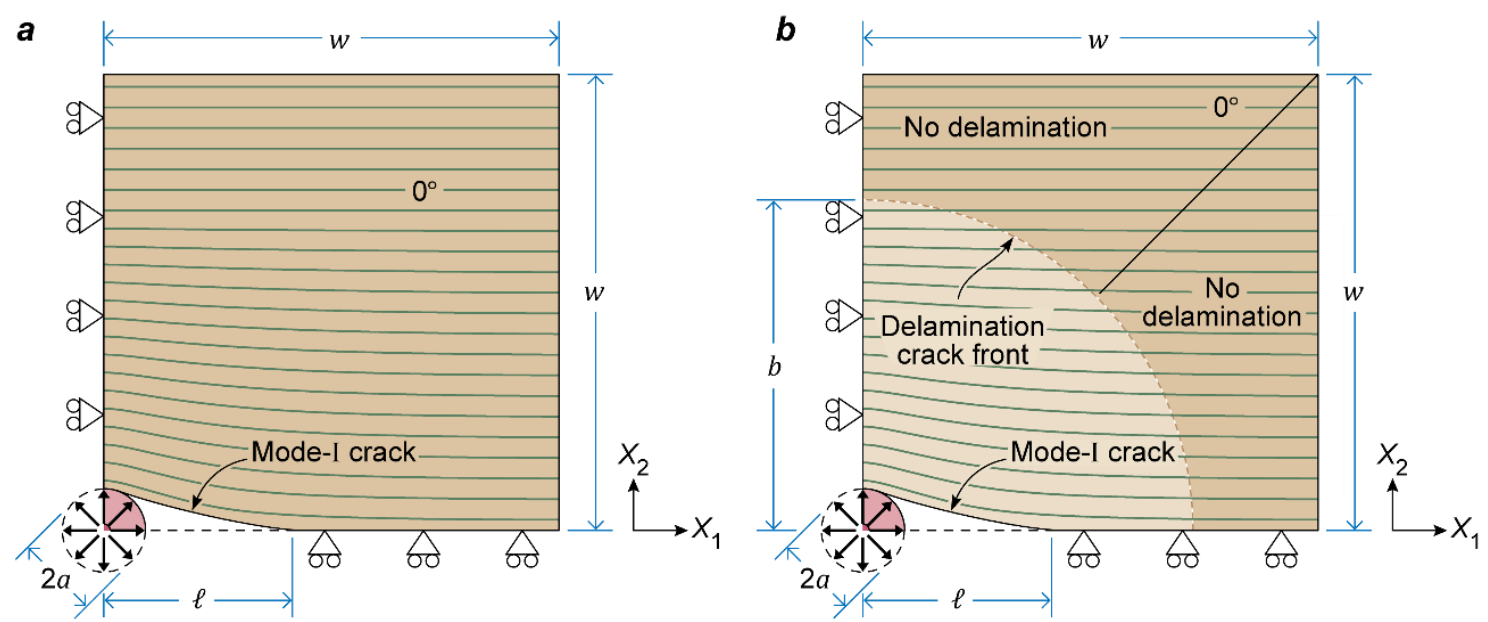

Figure 12: Sketches of the plane strain boundary value problems used to evaluate the energies (a) $U_{D}$ for the UD composite and (b) $V_{D}$ for the cross-ply composite, for inserting a circular punch of diameter $2 a$ into the composites. The global co-ordinate system and the fibre direction are indicated in each case.

The one quadrant of the plane strain slab of dimensions $w \times w$ (unit thickness in the $X_{3}$-direction) along with the imposed symmetry boundary conditions is sketched in Fig. 12a. A crack of length $2 \ell$ spans $-\ell \leq X_{1} \leq \ell$ and lies along $X_{2}=0$ and all calculations reported here used a slab of size $w=20 \ell$ (numerical experimentation confirmed that increasing the size of the block had a negligible effect on $U_{D}$ ). Loading was specified by expanding a circular body centred at $\left(X_{1}, X_{2}\right)=(0,0)$ from an initial radius $r=0^{+}$to $r=a$ at a strain rate $\dot{r} / r=$ $\dot{\gamma}_{0}$ with frictionless hard contact specified between the surface of the circular body and the crack flanks. The circular body is rigid in the sense that the loading imposed by the composite induces no deformation of the body. All calculations were performed using the commercial finite element (FE) package ABAQUS with the crystal plasticity constitutive model implemented as a user defined material model. The slab was meshed using 6-noded plane strain elements (CPE6 in the ABAQUS notation) and $U_{D}$ calculated as

$$
U_{D}=\int_{0}^{T} \int_{A} \dot{\varepsilon} d A d t^{\prime}
$$

Here $A$ denotes the undeformed domain of the composite with the circular body expanding from its initial radius $r=0^{+}$at time $t^{\prime}=0$ to $r=a$ at $t^{\prime}=T$. FE calculations were performed to determine $U_{D}$ in the range $2 \leq \ell / a \leq 6.2$ and the non-dimensional function $\mathcal{F}$ estimated via the definition (4.3). (FE predictions of $\mathcal{F}$ as a function of $\ell / a$ are included in Appendix B.) For a given toughness $J_{I C}$, the steady-state crack length $\ell_{s S}$ was then estimated via (4.5) which involved the numerical differentiation of $\mathcal{F}$ with respect to $\ell / a$ and the steadystate penetration pressure then follows from (4.4). We emphasize that consistent with the experiments, the expansion of the cylindrical insert for all values of $\ell / a$ investigated here was accommodated by elastic deformations and plastic shearing with negligible pull-in of the composite along the $X_{1}=w$ plane.

\footnotetext{
${ }^{2}$ Other loading paths such as indentation of a cylindrical punch of radius $a$ to a depth $a$ (Shergold and Fleck, 2004) were also investigated. These numerical results suggest that $U_{D}$ is relatively insensitive (to within $2 \%$ ) to the loading path.
} 


\subsection{Comparison of measurements and predictions}

Predictions of the steady-state penetration pressure $p_{s s}$ and the corresponding normalized crack lengths $\ell_{s S} / a$ are included in Fig. 6a along with the measurements for the choice of toughness $J_{I C}=10 \mathrm{~kJ} \mathrm{~m}^{-2}$. Excellent agreement is observed for both $p_{s s}$ and $\ell_{s S} / a$ over the range of punch diameters investigated here. In order to understand the dependence of these results on the choice of toughness we include in Fig. $6 \mathrm{~b}$ predictions of the non-dimensional steady-state penetration pressure $p_{s s} / \tau_{Y}$ and crack length $\ell_{s s} / a$ as function of the nondimensional toughness $J_{I C} /\left(\tau_{Y} a\right)$. With increasing toughness (or equivalently decreasing punch diameter $a$ ), the penetration pressure $p_{s s}$ increases. This is a result of a reduction in the relative cracking of the composite (i.e. decreasing $\ell_{s s} / a$ ) and the consequent increase in the deformation that the composite needs to undergo to allow the punch to penetrate. The penetration size effect is thus a result of this competition between deformation and cracking of the composite with the relevant material length scale characterised by $J_{I C} / \tau_{Y}$ : for the choice of $J_{I C}=10 \mathrm{~kJ} \mathrm{~m}^{-2}$ and $\tau_{Y}=2 \mathrm{MPa}, J_{I C} / \tau_{Y} \approx 5 \mathrm{~mm}$ and thus size effects are observed for millimetre size punch diameters.

One of the important outcomes of this analysis is that it provides an estimate of the mode-I inter-fibre splitting toughness $J_{I C}$ of the UHMWPE composites. No independent measurement of this toughness has been reported in the literature to-date, presumably because the design of traditional fracture specimens such as compact tension or bend specimens has proved elusive. To understand this recall that the plane strain plastic zone size at fracture scales as

$$
r_{p}=\frac{1}{3 \pi}\left(\frac{J_{I c}}{\tau_{Y}}\right)\left(\frac{E_{f}}{\tau_{Y}}\right)
$$

with $r_{p} \approx 21 \mathrm{~m}$ for the material parameter values used here with $J_{I C}=10 \mathrm{~kJ} \mathrm{~m}^{-2}$. Thus, a traditional fracture test such as a compact tension test (which involves applying a load remote from the crack tip) will result in plastic collapse of the specimen with no associated asymptotic $J$-field around the crack tip for any reasonable test specimen size. The penetration tests reported here, which involve wedging open of the crack, circumvent this issue by directly applying an opening displacement near the crack tip. This permits the generation of a $J$-field in a relatively small specimen. In fact, such wedging/cutting tests represent the only practical option for measuring the fracture toughness of soft materials (e.g. butter) and UHMWPE composites fall into this class of materials due to their extreme anisotropy with a very low shear strength. Nevertheless, the mode-I delamination toughness of cross-ply composites with the crack propagating at the interface between the $0^{\circ}$ and $90^{\circ}$ plies has been reported (Lässig et al., 2016) to be $\sim 400 \mathrm{~J} \mathrm{~m}^{-2}$. The intra-ply mode-I toughness inferred here is about 25 times higher at $J_{I C}=10 \mathrm{~kJ} \mathrm{~m}^{-2}$. To rationalise this, recall that Liu et al. (2014) and Attwood et al. (2015) observed that deformation of cross-ply UHMWPE composites occurs primarily by interlaminar shear rather than shearing within the plies. This suggests that intra-ply bonding of the matrix and the fibres is stronger than bonding between orthogonal plies. We thus argue that the higher intra-ply mode-I toughness is related to the stronger matrix/fibre bonding within plies compared to bonding between plies.

\section{Extension to the steady-state penetration of cross-ply composites}

We now proceed to extend the analysis of Section 4 to the steady-state penetration of the crossply composites with a ply thickness $t$. The X-ray image (Fig. 8) shows that penetration involves the formation of mode-I cracks along the fibre directions in the alternating $\left[0^{\circ} / 90^{\circ}\right]$ plies. The high stiffness of the plies in the fibre direction relative to their transverse stiffness implies that the deformations from the opening of inter-fibre cracks within the plies cannot be 
accommodated via compatible deformations. Rather the crack opening deformations are accommodated by inducing shear delaminations between the plies. Deep within the specimen these delaminations do not result in opening in the $X_{3}$-direction and thus the delaminations cannot be observed in X-ray images. Here we propose a model by assuming that delaminations form between every ply and these delamination zones are circles of radius $b$ centred on the axis of the punch; see Fig. 10b.

Consider the steady-state penetration of a cross-ply composite. The advance of the punch by the ply thickness $t$ results in the transformation of a single ply from its undeformed upstream state to its downstream state. In this downstream state the ply has a mode-I crack of length $2 \ell$ that accommodates the punch of radius $a$ and is delaminated from its adjacent ply over a circular zone of radius $b$. A work balance then dictates

$$
p_{s s}\left(\pi a^{2}\right) t=2 t \int_{0}^{\ell} J_{I} d \ell+2 \pi \int_{0}^{b} J_{d} b d b+V_{D},
$$

where $V_{D}$ is the energy required to open the crack of length $2 \ell$ to accommodate a cylinder of radius $a$ in a $0^{\circ}$ ply of thickness $t$ that has delaminated from its adjacent $90^{\circ}$ ply over a circular region of radius $b$ (Fig. 10b). Recalling that at steady-state the cracks are in a state of incipient crack advance, the mode-I and delamination toughnesses are related to $V_{D}$ via the usual energy release rate definitions as

$$
J_{I}=-\frac{1}{2 t} \frac{\partial V_{D}}{\partial \ell}
$$

and

$$
J_{d}=-\frac{1}{2 \pi b} \frac{\partial V_{D}}{\partial b}
$$

respectively. Here we emphasize that $J_{d}$ is a combined mode-II/III delamination toughness with no mode-I delamination component. Following Section 4.1 we express $V_{D}$ as

$$
V_{D} \equiv \tau_{Y} a^{2} t \mathcal{G}\left(\frac{\ell}{a}, \frac{b}{a}, \mathcal{M}\right)
$$

and again, assuming that the toughnesses are independent of crack advance (i.e. no R-curve effect) it follows that

$$
p_{s S}=\frac{2}{\pi}\left(\frac{J_{I C}}{a}\right)\left(\frac{\ell_{s S}}{a}\right)+\left(\frac{J_{d C}}{t}\right)\left(\frac{b}{a}\right)^{2}+\frac{\tau_{Y}}{\pi} \mathcal{G}\left(\frac{\ell_{s S}}{a}, \frac{b_{s S}}{a}, \mathcal{M}\right) .
$$

Here $J_{I C}$ and $J_{d C}$ are the intra-ply mode-I and delamination toughnesses, respectively and the crack lengths $\ell_{s s}$ and $b_{s s}$ during steady-state penetration satisfy the relations

and

$$
J_{I C}=-\left.\frac{\tau_{Y} a}{2} \frac{\partial \mathcal{G}}{\partial(\ell / a)}\right|_{\ell=\ell_{s S}},
$$

$$
J_{d C}=-\left.\frac{\tau_{Y} a t}{2 \pi b} \frac{\partial \mathcal{G}}{\partial(b / a)}\right|_{b=b_{s s}}
$$

respectively.

\subsection{Finite element calculation of the deformation energy}

The energy $V_{D}$ (or equivalently $\mathcal{G}$ ) was calculated via FE calculations in a manner analogous to that described for the UD composite in Section 4.3. The main difference lies in simulating the bonded and delaminated parts of the plies of the cross-ply composite. This is accomplished by modelling a single ply of thickness $t$ with appropriately imposed boundary conditions and 
constraints. Here we explain these constraints by considering a single $0^{\circ}$ ply although a completely equivalent analysis is possible for a $90^{\circ}$ ply.

We wish to calculate $V_{D}$ for penetration by a punch of radius $a$ with a mode-I crack of length $2 \ell$ and a circular delaminated zone of radius $b$ centred at the axis of the punch. Using symmetry considerations, we model a quarter segment of a single $0^{\circ}$ ply of thickness $t$ as shown in Fig. 12b such that the mode-I crack lies along $X_{2}=0$ over the region $-\ell \leq X_{1} \leq \ell$. The delaminated zone is a circle of radius $b \geq \ell$ centred at $\left(X_{1}, X_{2}\right)=(0,0)$ with deformation of the ply restricted to the $X_{1}-X_{2}$ plane, i.e. plane strain condition is imposed. Loading is specified by the expansion of a circular body centred at $(0,0)$ as explained in Section 4.3 and here we focus on explaining the application of constraints to simulate the delaminated and bonded portions of the ply.

In the delaminated region $\sqrt{X_{1}^{2}+X_{2}^{2}}<b$, there is no deformation constraint imposed by the adjacent $90^{\circ}$ ply and hence no further constraints are specified on the material point displacement $\left(u_{1}, u_{2}\right)$ in this region. In the bonded region, $\sqrt{X_{1}^{2}+X_{2}^{2}} \geq b$, the adjacent $90^{\circ}$ plies impose constraints on the deformation of the $0^{\circ}$ ply. To understand these constraints, recall that the $90^{\circ}$ and $0^{\circ}$ plies are identical except rotated $90^{\circ}$ with respect to each other about the $X_{3}$-axis. Thus, in the bonded zone $\sqrt{X_{1}^{2}+X_{2}^{2}} \geq b$, the displacements in the region $\left(X_{1}, X_{2}\right)$ with $X_{1} \leq X_{2}$ are related to the displacements in the region $\left(X_{1}, X_{2}\right)$ with $X_{1}>X_{2}$ as

$$
\begin{aligned}
& u_{1}\left(X_{1}, X_{2}\right)=u_{2}\left(X_{2}, X_{1}\right) \\
& u_{2}\left(X_{1}, X_{2}\right)=u_{1}\left(X_{2}, X_{1}\right) .
\end{aligned}
$$

The energy $V_{D}$ (or equivalently the non-dimensional function $\mathcal{G}$ ) was evaluated via $\mathrm{FE}$ calculations using the material properties detailed in Section 4.2 and the constraints on deformation described above. Calculations were performed for crack lengths ranging $2 \leq$ $\ell / a \leq 5.4$ and $12 \leq b / a \leq 29$. (FE predictions of $\mathcal{G}(\ell / a, b / a, \mathcal{M}$ ) are included in Appendix B.) The steady-state crack length $\ell_{s s}$ and $b_{s s}$ then follow from Eqs. (5.6) and (5.7) with appropriate choices of the toughnesses $J_{I C}$ and $J_{d C}$.

\subsection{Comparison of measurements and predictions}

Predictions of the steady-state pressure $p_{s s}$ and associated normalized mode-I crack lengths $\ell_{s s} / a$ are included in Fig. $9 \mathrm{a}$ as a function of the punch diameter $2 a$ for the cross-ply composite with ply thickness $t=60 \mu \mathrm{m}$. The mode-I intra-ply toughness is taken to be equal to that estimated from the UD penetration measurements and predictions (i.e. $J_{I C}=10 \mathrm{~kJ} \mathrm{~m}^{-2}$ ) while the delamination toughness was set to $J_{d C}=25 \mathrm{~J} \mathrm{~m}^{-2}$ so as to obtain the high level of agreement between predictions and measurements seen in Fig. 9a. The high penetration pressure $p_{s s}$ of the cross-ply composites relative to the UD composites (the predictions of $p_{s s}$ for the UD composites from Fig. 6a are included in Fig. 9a) is due to a combination of the constrained deformation of the plies in the non-delaminated region and the additional dissipation resulting from the delamination of the plies around the penetrator. Moreover, over the range of punch diameters investigated in the experimental study, the size effect on the steady-state penetration pressure is relatively small. However, the predictions in Fig. 9a indicate that similar to the UD composites, $p_{s s}$ rises sharply for $2 a<1 \mathrm{~mm}$. The corresponding predictions for cross-ply composites penetrated by a punch of diameter $2 a=$ $2 \mathrm{~mm}$ are included in Fig. $9 \mathrm{~b}$ as a function of the ply thickness $t$ : the good agreement with the measurements confirms the fidelity of the model and that the $J_{I C}$ and $J_{d C}$ values inferred here 
give good predictions over a range of composite types (UD and cross-ply), punch diameters and ply thicknesses of the cross-ply composites.

Measurements of the size $b_{s S}$ of the delamination zone are not available as discussed above. Thus, while comparisons between observations and predictions of $b_{s s}$ are not possible it is instructive to discuss predictions of the size of delamination zone. Predictions of $b_{s s} / a$ as well as $\ell_{s s} / a$ are included in Fig. 13 as a function of the normalized delamination toughness $J_{d C} /\left(\tau_{Y} t\right)$ for selected values of the normalized mode-I toughness $J_{I C} /\left(\tau_{Y} a\right)$. These results are restricted to a toughness ratio $J_{I C} / J_{d C}=400$ corresponding to $J_{I C}=10 \mathrm{~kJ} \mathrm{~m}^{-2}$ and $J_{d C}=$ $25 \mathrm{~J} \mathrm{~m}^{-2}$. While both $b_{s s} / a$ and $\ell_{s s} / a$ decrease with increasing $J_{d c}$ (or equivalently decreasing ply thickness $t$ ), there are three crucial differences: (i) the delamination zone as parameterised by $b_{s s} / a$ extends out much further than the mode-I cracks, i.e. $b_{s s} / a \gg \ell_{s s} / a$; (ii) the size of the delaminated zone $b_{s s} / a$ depends strongly on $J_{d C} /\left(\tau_{Y} t\right)$ while the mode-I crack lengths display only a mild dependence on $J_{d C} /\left(\tau_{Y} t\right)$ and (iii) $\ell_{s s} / a$ is sensitive to the punch diameter as parameterised by $J_{I C} /\left(\tau_{Y} a\right)$ while this parameter has no influence on $b_{s S} / a$ (at-least to within the numerical accuracy of the calculations). Thus, we argue that the delamination zone sizes are mainly set by the delamination toughness and the ply thickness.

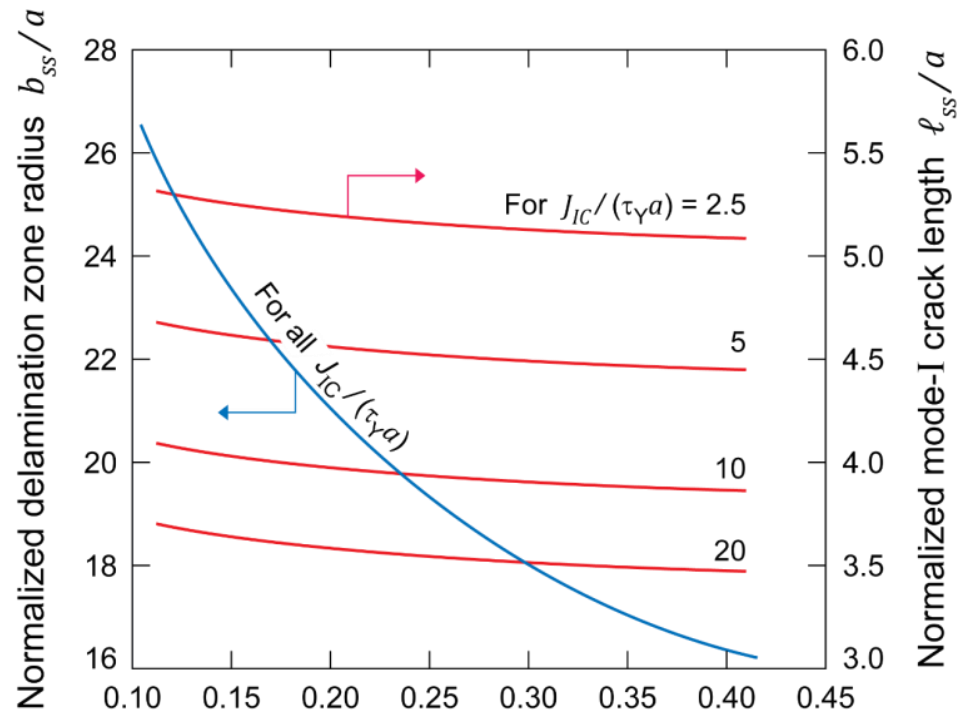

Normalized delamination toughness $J_{d C} /\left(\tau_{Y} t\right)$

Figure 13: Predictions of the normalized delamination zone size $b_{s s} / a$ and the corresponding normalized mode-I crack lengths $\ell_{s s} / a$ as a function of the normalized delamination toughness $J_{d C} /\left(\tau_{Y} t\right)$ for cross-ply composites with a toughness ratio $J_{I C} / J_{d C}=400$. Results are shown for selected values of the mode-I toughness $J_{I C} /\left(\tau_{Y} a\right)$.

The relatively large delamination zone size of $b_{s s} / a \approx 21$ for the $t=60 \mu \mathrm{m}$ cross-ply composite implies that specimens of size $L>42 \mathrm{~mm}$ are required to ensure that the delamination zone does not extend to the edge of a specimen penetrated centrally by a $2 a=$ $2 \mathrm{~mm}$ punch. In fact, penetration experiments conducted on $L=40 \mathrm{~mm}$ specimens of the $t=$ $60 \mu \mathrm{m}$ cross-ply composite displayed delaminations extending to the edge of the specimen. This indirectly confirmed both the existence of the delamination zones as well as the quantitative fidelity of the predictions.

Similar to the measurement of $J_{I C}$ inferred from the penetration experiments on the UD composites, the penetration measurements on the cross-ply composites provide an estimate of the combined mode-II/III delamination toughness $J_{d C}$. Again, there are no reported 
independent measurements of this toughness. The predictions and measurements reported here suggest that $J_{d C} \ll J_{I C}$. Thus, consistent with the observations made in Section 4.4 on the inferior inter-laminar properties of UHMWPE composites we surmise that this difference in the magnitude of the two toughnesses is related to the poor inter-ply bonding between the matrix and the fibres.
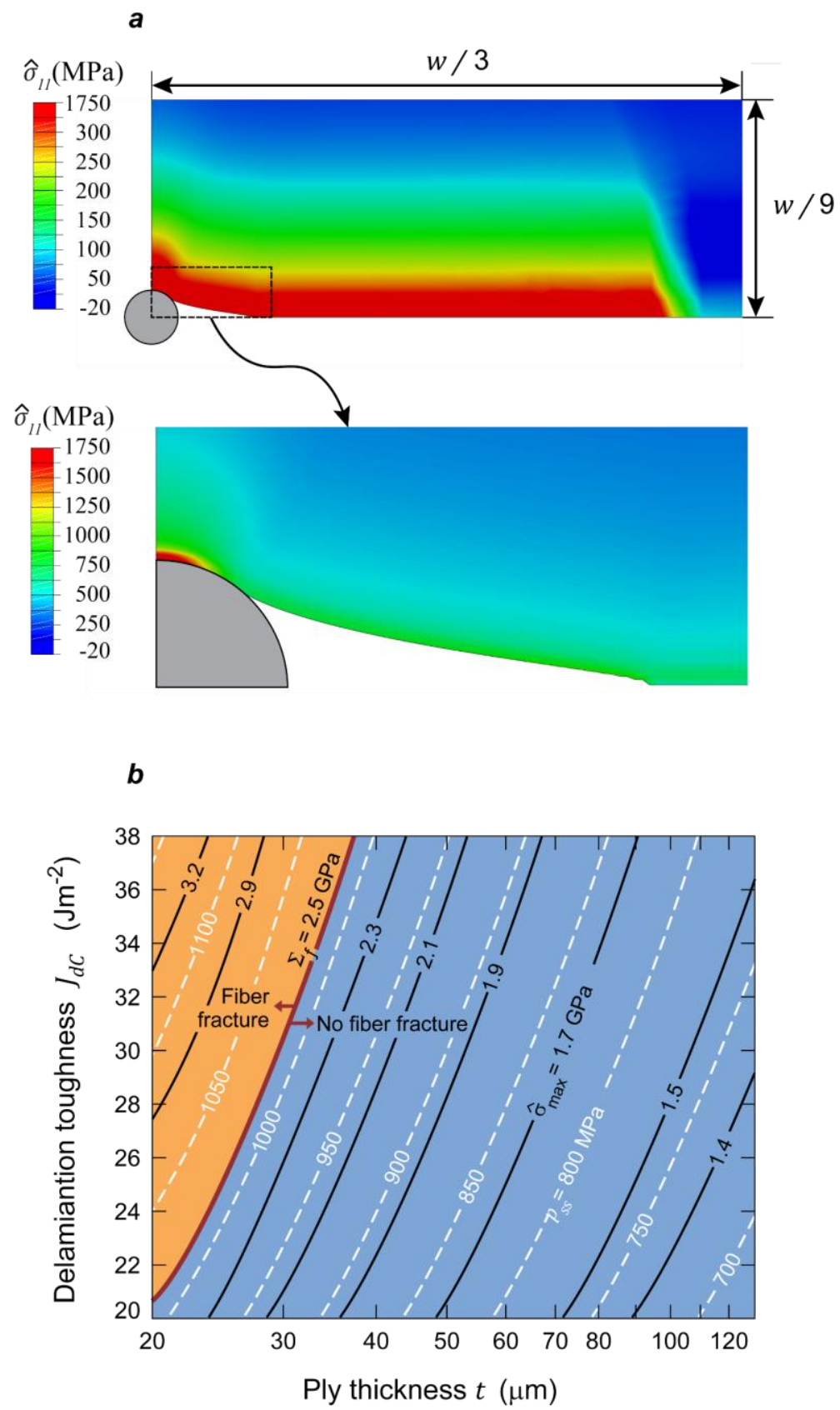

Figure 14: (a) FE predictions of the corotational stress $\hat{\sigma}_{11}$ around the punch in a $0^{\circ}$ ply within a $t=60 \mu \mathrm{m}$ cross-ply composite penetrated by a $2 a=2 \mathrm{~mm}$ punch, with normalized mode-I crack length $\ell / a=4$ and delamination zone radius $b / a=21$. Note that the magnified view is shown with a different stress scale to illustrate the highly stressed regions more clearly. (b) A deformation mechanism map with axes of the delamination toughness $J_{d C}$ and ply thickness $t$ for penetration by a $2 a=2 \mathrm{~mm}$ punch. Contours of the steady-state penetration pressure $p_{s S}$ and maximum ply stress $\hat{\sigma}_{\max }$ are included with the boundary demarcating the region of fibre fracture marked for the choice of ply strength $\Sigma_{f}=2.5 \mathrm{GPa}$. 


\section{Design maps for penetration resistance}

The high penetration resistance of the cross-ply UHMWPE composites makes them attractive candidates for use in systems such as vests for protection against knife attacks. With the development of new higher strength UHMWPE fibres as well as methods to manufacture composites with specified ply thicknesses, an immediate question that arises is: what is the optimal combination of material and microstructural parameters to maximise penetration resistance? With this in mind we will develop a design methodology to optimise the ply thickness for a given tensile strength $\Sigma_{f}$ of the ply in the fibre direction and a delamination toughness $J_{d c}$. Unless otherwise specified, the design maps in this section use all materials properties fixed at the values specified in Section 4.2 with $J_{I C}=10 \mathrm{~kJ} \mathrm{~m}^{-2}$ and $J_{d C}=$ $25 \mathrm{~J} \mathrm{~m}^{-2}$.

\subsection{Deformation mechanism map}

The UHMWPE composite conforms around the penetrating cylindrical punch as seen in Fig. 5. This deformation is expected to generate the highest fibre stresses at or near the contact between the composite and the cylindrical surface of the punch. In all the measurements reported here, these fibre stresses were insufficient to result in fibre fracture although it is conceivable that penetration of a composite with a sufficiently high toughness and/or sufficiently thin plies will result in fibre fracture. Here we develop a deformation mechanism map to investigate the range of material parameters where fibre fracture will be triggered by penetration.

In order to evaluate the occurrence of fibre fracture we define a co-rotational stress in the intermediate configuration

$$
\hat{\sigma}_{i j}=R_{m i}^{*} \sigma_{m n} R_{n j}^{*}
$$

such that $\hat{\sigma}_{11}$ gives the true ply stress in the local fibre direction in a $0^{\circ}$ ply with $R_{i j}^{*}$ denoting the proper orthogonal rotation tensor of $F_{i j}^{*}$. FE predictions of $\hat{\sigma}_{11}$ in a $0^{\circ}$ ply within a crossply composite penetrated by a $2 a=2 \mathrm{~mm}$ punch is shown in Fig. 14a for normalized mode-I crack length $\ell / a=4$ and delamination zone radius $b / a=21$. Recall that the model is only applicable for steady-state penetration and hence these predictions only pertain to $0^{\circ}$ plies which are both far downstream from the punch tip and deep within the specimen. The maximum value of $\hat{\sigma}_{11}$, labelled $\hat{\sigma}_{\text {max }}$ occurs at the contact between the composite and the punch along the $X_{1}=0$ symmetry line. Fibre fracture will occur if $\hat{\sigma}_{\text {max }}$ exceeds a material critical value $\Sigma_{f}\left(\Sigma_{f}=v_{f} \sigma_{f}\right.$ for UHMWPE composites comprising a volume fraction $v_{f}$ of fibres with a tensile strength $\sigma_{f}$ ) and thus we proceed to investigate the dependence of $\hat{\sigma}_{\text {max }}$ on ply thickness $t$ and delamination toughness $J_{d C}$ for a cross-ply composite penetrated by a punch of diameter $2 a=2 \mathrm{~mm}$. All other material parameters are held fixed at their reference values.

A deformation mechanism map with axes $J_{d C}$ and ply thickness $t$ is plotted in Fig. 14b for penetration by the $2 a=2 \mathrm{~mm}$ punch. Contours of both the maximum ply stress $\hat{\sigma}_{\max }$ and the steady-state penetration pressure $p_{s s}$ are included in the map. These contours are approximately parallel to each other indicating that both these metrics have a similar dependence on $\left(J_{d C}, t\right)$ with $\hat{\sigma}_{\max }$ as well as $p_{s s}$ increasing with increasing $J_{d C}$ and decreasing $t$. The contour $\hat{\sigma}_{\max }=\Sigma_{f}$ demarcates the regime where fibre fracture is expected to occur for the choice $\Sigma_{f}=2.5 \mathrm{GPa}$ (This choice is based on the strength $\sigma_{f}=3 \mathrm{GPa}$ of the SK76 UHMWPE fibres and a fibre volume fraction $v_{f}=83 \%$ in the composites investigated here.) 
The composites investigated here have a delamination toughness $J_{d C}=25 \mathrm{~J} \mathrm{~m}^{-2}$ and the map in Fig. 14b suggests that fibre fracture in this class of composites will occur for composites with ply thickness $t<25 \mu \mathrm{m}$.

\subsection{Optimal designs}

Suppose we wish to maximise the penetration resistance of the cross-ply composite as parameterised by $p_{s s}$ for a given ply strength $\Sigma_{f}$. Since the contours of $p_{s s}$ and $\hat{\sigma}_{\text {max }}$ are parallel to each other in Fig. 14b, this optimisation reduces to determining the combination of $J_{d C}$ and $t$ such that the maximum ply stress $\hat{\sigma}_{\max }=\Sigma_{f}$. Moreover, this also implies that the maximum penetration pressure $p_{S S}^{\max }$ is a unique function of $\Sigma_{f}$. This dependence of $p_{S S}^{\max }$ on $\Sigma_{f}$ for the $2 a=2 \mathrm{~mm}$ diameter punch is plotted in Fig. 15a from the design map in Fig. 14b, i.e. material properties listed in Section 4.2 with $J_{I C}=10 \mathrm{~kJ} \mathrm{~m}^{-2}$. We observe that increasing the ply strength from $\Sigma_{f}=2.5 \mathrm{GPa}$ to $3 \mathrm{GPa}$ increases $p_{s s}^{\max }$ from approximately $1 \mathrm{GPa}$ to about $1.1 \mathrm{GPa}$.
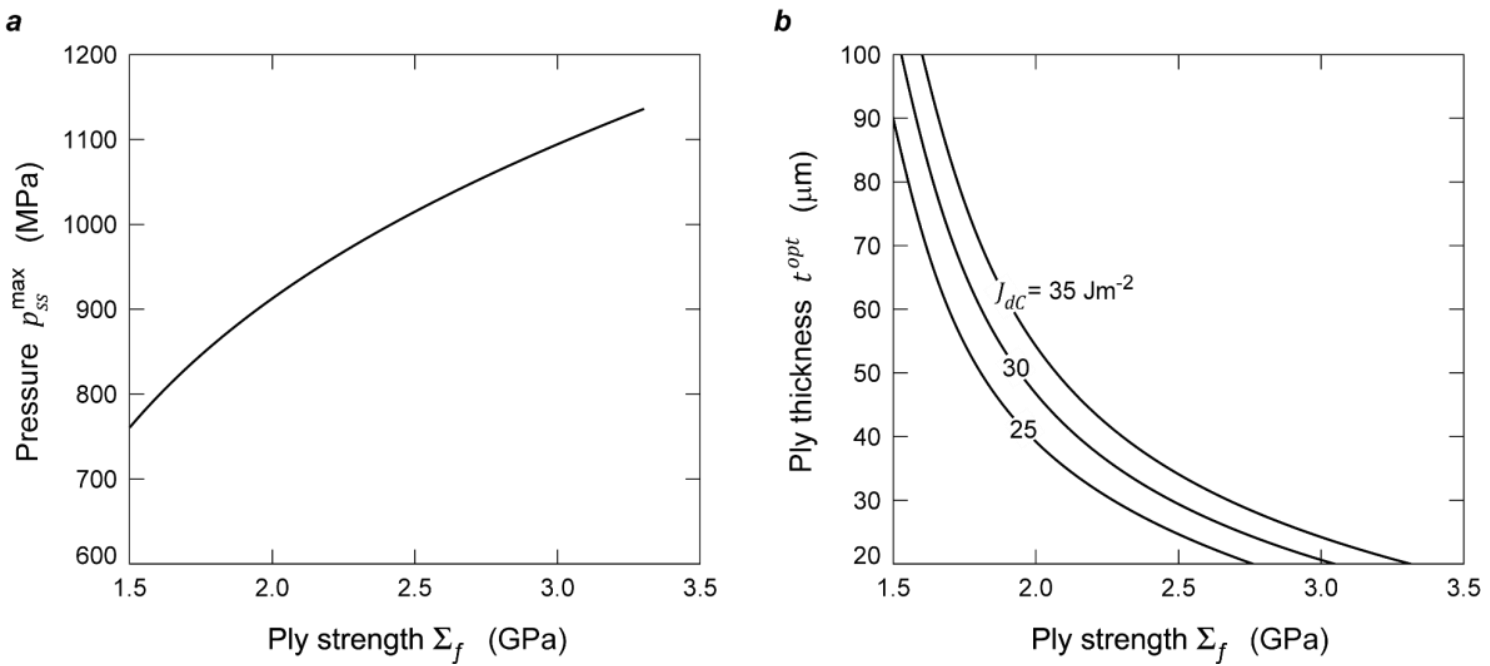

Figure 15: Predictions of (a) the maximum steady-state penetration pressure $p_{s s}^{\max }$ as a function of the ply strength $\Sigma_{f}$ of the cross-ply composite. (b) The corresponding optimal ply thickness $t^{\text {opt }}$ as a function of $\Sigma_{f}$ for three selected values of the delamination toughness $J_{d c}$.

Optimal designs as characterised by $\left(J_{d C}^{\mathrm{opt}}, t^{\mathrm{opt}}\right.$ ) for a given $\Sigma_{f}$ (all have an equal penetration resistance $p_{S S}^{\max }$ as plotted in Fig. 15a) can immediately be obtained by following the contour $\hat{\sigma}_{\text {max }}=\Sigma_{f}$ in Fig. 14a. However, it is typically difficult to alter the ply strength (set mainly by the fibre strength) and the delamination toughness but relatively straightforward to change the ply thickness. Thus, a practical optimisation problem is to determine the optimal ply thickness $t^{\text {opt }}$ for a given combination of $J_{d C}$ and $\Sigma_{f}$. Predictions of this optimal ply thickness are included in Fig. 15b as a function of $\Sigma_{f}$ for three selected values of $J_{d c}$. With increasing ply strength $\Sigma_{f}$, the ply thickness $t^{\text {opt }}$ required to attain the maximum penetration pressure $p_{S S}^{\max }$ decreases. In fact, for $\Sigma_{f}>2.75 \mathrm{GPa}$, the optimum ply thickness drops below $20 \mu \mathrm{m}$ for $J_{d C}=$ $25 \mathrm{~J} \mathrm{~m}^{-2}$. Composites with such thin plies are not only challenging to manufacture but also impractical as they have a thickness on the order of the fibre diameter. The results in Fig. 15 suggest that $t^{\text {opt }}$ increases with increasing $J_{d C}$ for a given ply strength, i.e. increasing the delamination toughness will permit optimal performance for composites with practical ply thicknesses. 


\section{Concluding remarks}

The penetration of fibre reinforced ultra-high molecular weight polyethylene (UHMWPE) composites by sharp-tipped cylindrical punches was investigated through both measurements and associated micro-mechanical models. The main findings can be summarised as follows:

(i) Penetration occurred with no fibre fracture and the penetration pressure increased with decreasing punch diameter for both UD and cross-ply composites. This size dependence was observed for punch diameters on the millimetre length scale and thus much stronger than the micron scale size dependence of the indentation/penetration response of metals.

(ii) The penetration pressure also increased with decreasing ply thickness of the crossply composites. In both UD and cross-ply composites, the sharp-tipped punch penetrates by the formation of mode-I cracks along the fibre directions, followed by the wedging open of the crack by the advancing punch. These mode-I cracks form in orthogonal directions in the adjacent $0^{\circ}$ and $90^{\circ}$ plies in the cross-ply composite. The ensuing incompatible deformation between these adjacent plies induces delamination around the penetrator.

(iii) A combination of the dissipation due to delamination and the constraint imposed on the deformation of each ply by the alternating $0^{\circ}$ and $90^{\circ}$ plies results in a penetration pressure on the order of $1 \mathrm{GPa}$ for the cross-ply composites and is approximately 3 times larger than that of a UD composite penetrated by a punch of equal diameter.

(iv) The micro-mechanical models suggest that the competition between the deformation of the composite by wedging open of the crack and the extension of the mode-I and delamination cracks results in the observed size dependence of the penetration pressure.

(v) The models were used to develop design maps for the penetration resistance of cross-ply composites and to infer composite designs that maximise the penetration resistance for a given fibre strength. In particular, these calculations suggest that increasing the delamination toughness permits optimum performance for composites with thicker plies that are more practical from a manufacturing perspective.

The penetration measurements and models reported here have provided estimates of the modeI inter-fibre splitting toughness $J_{I C}$ and the combined mode-II/III delamination toughness $J_{d C}$. The large mode-I fracture toughness and the low shear strength of UHMWPE composites make the design of traditional fracture toughness tests (e.g. compact tension) very difficult and hence to-date no measurements of these toughnesses have been reported in the literature. In fact, our estimates suggest that $J_{I C} / J_{d C}=400$ with $J_{I C}=10 \mathrm{~kJ} \mathrm{~m}^{-2}$. Most UHMWPE composite penetration experiments involve blunt penetrators with continued penetration occurring via fibre fracture. The high penetration resistance in such circumstances is attributed to the high fibre strength. By contrast, penetration by sharp-tipped punches involves no fibre fracture, and we argue that the high penetration resistance in such cases is to a large extent due to the high toughnesses of these composites.

\section{Acknowledgements}

The work was funded by the Defence Advanced Research Projects Agency (DARPA) under grant number W91CRB-11-1-0005. We are grateful to DSM for providing the Dyneema ${ }^{\circledR}$ composites. VSD and HNGW would like to congratulate Prof. Norman Fleck on the occasion 
of his $60^{\text {th }}$ birthday and thank him for the many years of friendship and inspiration that have characterized our collaborations.

\section{Appendix A: Protocol for estimating crack lengths in the cross-ply specimens from plain radiographs}

$\mathrm{X}$-ray tomographic imaging of the cross-ply composites was not feasible as the X-ray opaque Tungsten-Carbide/Cobalt punches cast a large shadow. We thus inferred the sizes of the modeI intra-ply cracks in the cross-ply composites from plain radiographs. In plain radiographs, geometric magnification results from the fact that the detector is further away from the source than the object and here we explain the methodology used to determine the radiographic magnification factor and hence extract the mode-I crack lengths from plain radiographs such as the image shown in Fig. 8.

Consider the imaging of a cross-ply composite along with the penetrator. The plain radiograph is taken by shining an X-ray source in the $X_{3}$-direction with the rear of the punch nearest to the source as sketched in Fig. A1. For the sake of simplicity, we neglect the finite size of the focal spot of the X-ray source and hence ignore the resulting unsharpness in the image on the detector plane. The radiographic magnification is clearly seen in Fig. Ala where the cylindrical punch of diameter $2 a$ appears as a shadow of diameter $2 \mathcal{A}$ on the detector plane. We wish to determine the mode-I crack size $2 \ell_{s s}$ from its image of length $2 \mathcal{L}$ on the detector plane. With $\mathcal{H}$ denoting the source-detector distance, geometric constructions based on similar triangles give

$$
\frac{\ell_{s S}}{\mathcal{L}}=\frac{s+h_{1}+h_{2}}{\mathcal{H}} \text { and } \frac{a}{\mathcal{A}}=\frac{s}{\mathcal{H}}
$$

where $s$ is the distance from the source to the rear of the punch while $h_{1}$ and $h_{2}$ are the punch lengths outside and within the specimen such that $h_{1}+h_{2}$ is the total length of the punch (here we assume that the mode-I crack attains its steady-state length at $\xi \approx a \tan \alpha$ ). Combining the equations in (A1) we can eliminate $\mathcal{H}$ to obtain

$$
\frac{\ell_{s S}}{a}=\left(\frac{s+h_{1}+h_{2}}{a}\right)\left(\frac{\mathcal{L}}{\mathcal{A}}\right) \text {. }
$$

A direct measure of $s$ is not available from the X-ray setup and hence an indirect method was used to estimate $s$. An X-ray opaque circular calibration ring of external diameter $2 c$ was placed on the flat surface of the specimen. This ring casts a shadow of diameter $2 \mathcal{C}$ (Fig. A1b) on the detector plane and again using similar triangles we have

$$
\frac{c}{\mathcal{C}}=\frac{s+h_{1}}{\mathcal{H}}
$$

which upon combining with (A1) gives

$$
s=\left(\frac{a / \mathcal{A}}{c / \mathcal{C}-a / \mathcal{A}}\right) h_{1} .
$$

Equations (A2) and (A4) then directly provides an estimate of $\ell_{s s}$ : measurements of $\mathcal{L}, \mathcal{A}$ and $\mathcal{C}$ are directly available from measurements from the radiograph while $a$ and $c$ are known sizes of the punch and the ring. The length $h_{1}$ is measured from the penetrated specimen while $h_{2}$ is the residual length of the punch. 

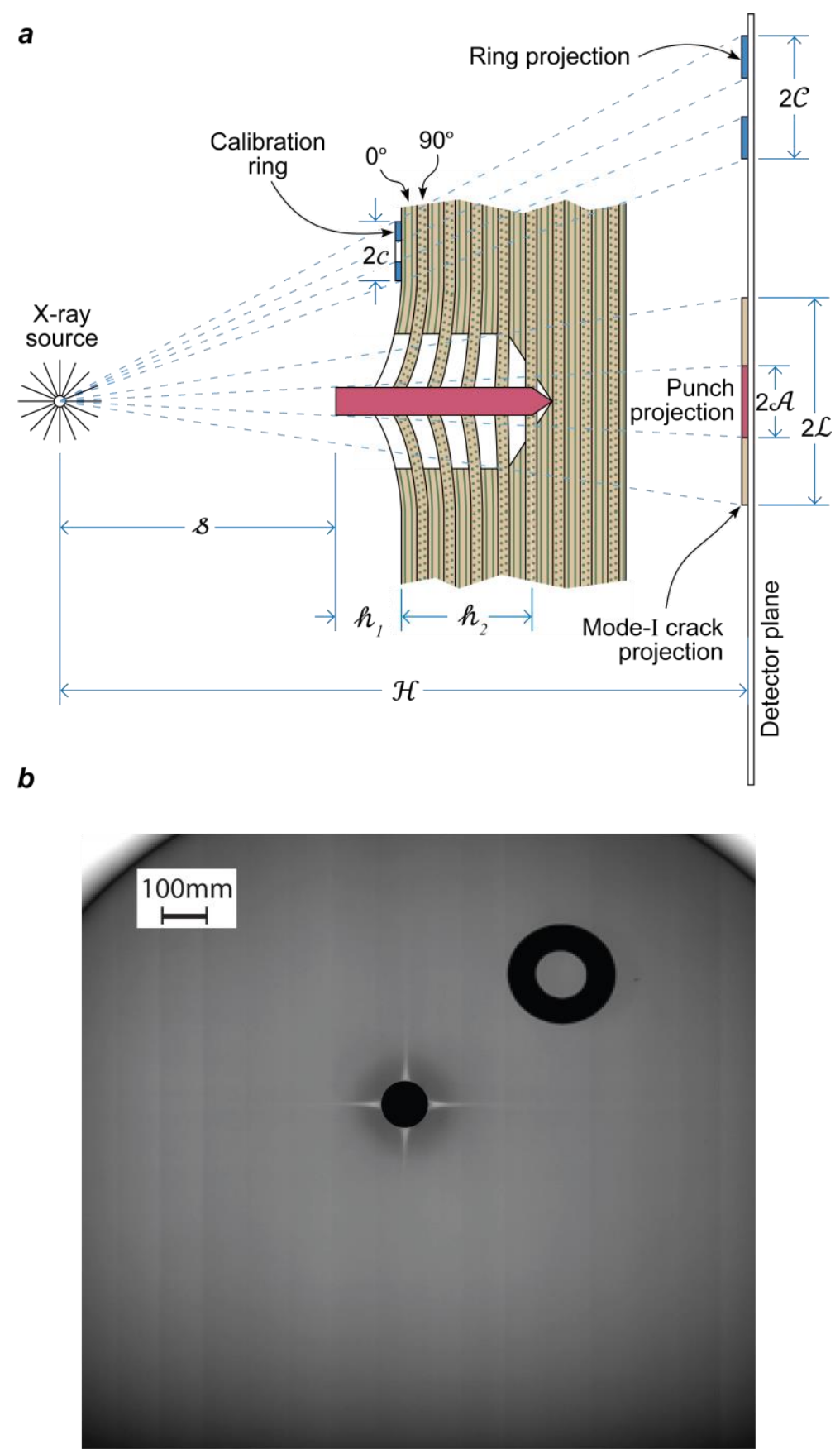

Figure A1: (a) Sketch of the setup for a plain radiograph of the penetrated cross-ply composite with the symbols of key dimensions marked. (b) A plane radiograph of a $t=60 \mu \mathrm{m}$ cross-ply composite penetrated by a $2 a=3 \mathrm{~mm}$ punch. The radiograph shows the entire image on the detector plane including the image of the calibration ring. The scale bar shows the size of the image on the detector plane.

\section{Appendix B: FE predictions of the non-dimensional deformation energies $\mathcal{F}$ and $\mathcal{G}$}

FE predictions of the non-dimensional deformation energy $\mathcal{F}$ for the UD composite is included in Fig. B1a for non-dimensional mode-I crack lengths in the range $2 \leq \ell / a \leq 5$ while the equivalent energy $\mathcal{G}$ for the cross-ply is plotted in Fig. B1b as a function of $\ell / a$ for selected values of the non-dimensional delamination zone size $b / a$. Given the toughnesses, $J_{I C}$ and $J_{d C}$, the derivatives of these functions directly provide the steady-state crack sizes and the penetration pressures as detailed in Section 4. 

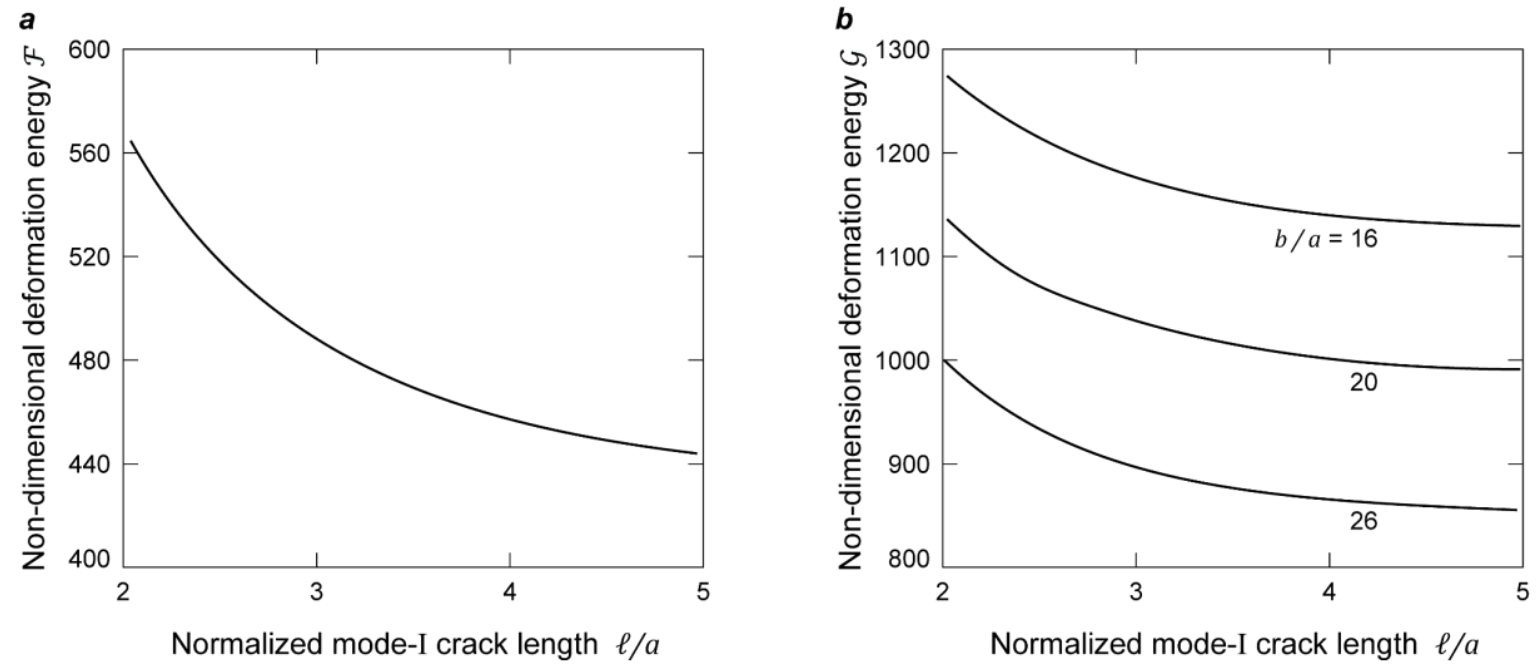

Figure B1: FE predictions of the non-dimensional deformation energies (a) $\mathcal{F}$ for the UD composite and (b) $\mathcal{G}$ for the cross-ply composite as a function of the normalized mode-I crack length $\ell / a$. In (b) $\mathcal{G}$ is shown for selected values of the normalized delamination zone radius $b / a$.

\section{References}

Anderson, T.L., 2005. Fracture Mechanics: Fundamentals and Applications, $3^{\text {rd }}$ Edition, CRC Press.

Asaro, R.J., 1983. Micromechanics of Crystals and Polycrystals. Adv. Appl. Mech. 23, 1-115. https://doi.org/10.1016/S0065-2156(08)70242-4

Asaro, R.J., Needleman, A., 1985. Overview no. 42 Texture development and strain hardening in rate dependent polycrystals. Acta Metall. 33, 923-953. https://doi.org/10.1016/0001-6160(85)901889

Attwood, J.P., Khaderi, S.N., Karthikeyan, K., Fleck, N.A., O'Masta, M.R., Wadley, H.N.G., Deshpande, V.S., 2014. The out-of-plane compressive response of Dyneema composites. J. Mech. Phys. Solids 70, 200-226. https://doi.org/10.1016/j.jmps.2014.05.017

Attwood, J.P., Russell, B.P., Wadley, H.N.G., Deshpande, V.S., 2016. Mechanisms of the penetration of ultra-high molecular weight polyethylene composite beams. Int. J. Impact Eng. 93, 153-165. https://doi.org/10.1016/j.ijimpeng.2016.02.010

Bao, L., Sato, S., Morikawa, H., Soma, S., 2016. Improving stab-resistant textile materials with a nonwoven fabric structure. J. Text. Eng. 62, 37-42. https://doi.org/10.4188/jte.62.37

Bilisik, K., 2017. Two-dimensional (2D) fabrics and three-dimensional (3D) preforms for ballistic and stabbing protection: A review. Text. Res. J. 87, 2275-2304. https://doi.org/10.1177/0040517516669075

Cheeseman, B.A., Bogetti, T.A., 2003. Ballistic impact into fabric and compliant composite laminates. Compos. Struct. 61, 161-173. https://doi.org/10.1016/S0263-8223(03)00029-1

Chocron, S., Nicholls, A.E., Brill, A., Malka, A., Namir, T., Havazelet, D., Werff, H. van der, Heisserer, U., Walker, J.D., 2014. Modeling unidirectional composites by bundling fibers into strips with experimental determination of shear and compression properties at high pressures. Compos. Sci. Technol. 101, 32-40. https://doi.org/10.1016/j.compscitech.2014.06.016

Cunniff, P.M., 1999. Dimensionless Parameters for Optimization of Textile-Based Body Armor Systems. 18th Int. Symp. Ballist. 1303-1310.

Decker, M.J., Halbach, C.J., Nam, C.H., Wagner, N.J., Wetzel, E.D., 2007. Stab resistance of shear thickening fluid (STF)-treated fabrics. Compos. Sci. Technol. 67, 565-578. https://doi.org/10.1016/j.compscitech.2006.08.007

Govaert, L.E., Peijs, T., 1995. Tensile strength and work of fracture of oriented polyethylene fibre. Polymer (Guildf). 36, 4425-4431. https://doi.org/10.1016/0032-3861(95)96848-3

Grujicic, M., Arakere, G., He, T., Bell, W.C., Cheeseman, B.A., Yen, C.F., Scott, B., 2008. A ballistic material model for cross-plied unidirectional ultra-high molecular-weight polyethylene fiber- 
reinforced armor-grade composites. Mater. Sci. Eng. A 498, 231-241. https://doi.org/10.1016/j.msea.2008.07.056

Heisserer, U., Werff, H. Van Der, Hendrix, J., 2013. Ballistic depth of penetration studies in Dyneema ${ }^{\circledR}$ composites, in: Proceedings of the 27th International Symposium on Ballistics, Vol. 2. Freiburg, Germany. pp. 1936-1943.

Hill, R., Rice, J.R., 1972. Constitutive analysis of elastic-plastic crystals at arbitrary strain. J. Mech. Phys. Solids 20, 401-413. https://doi.org/10.1016/0022-5096(72)90017-8

Iannucci, L., Pope, D., 2011. High velocity impact and armour design. Express Polym. Lett. 5, 262272. https://doi.org/10.3144/expresspolymlett.2011.26

Karthikeyan, K., Russell, B.P., 2014. Polyethylene ballistic laminates: Failure mechanics and interface effect. Mater. Des. 63, 115-125. https://doi.org/10.1016/j.matdes.2014.05.069

Karthikeyan, K., Russell, B.P., Fleck, N.A., O’Masta, M., Wadley, H.N.G., Deshpande, V.S., 2013. The soft impact response of composite laminate beams. Int. J. Impact Eng. 60, 24-36. https://doi.org/10.1016/j.ijimpeng.2013.04.002

Kim, S.J., Goo, N.S., 1997. Dynamic contact responses of laminated composite plates according to the impactor's shapes. Comput. Struct. 65, 83-90. https://doi.org/10.1016/S0045-7949(95)00408-4

Lässig, T.R., Nolte, F., Riedel, W., May, M., 2016. An assessment of experimental techniques for measuring the mode I fracture toughness of uhmw-pe composites. Proc. 17th Eur. Conf. Compos. Mater. ECCM17 26-30.

Li, W., Xiong, D., Zhao, X., Sun, L., Liu, J., 2016. Dynamic stab resistance of ultra-high molecular weight polyethylene fabric impregnated with shear thickening fluid. Mater. Des. 102, 162-167. https://doi.org/10.1016/j.matdes.2016.04.006

Liu, G., Thouless, M.D., Deshpande, V.S., Fleck, N.A., 2014. Collapse of a composite beam made from ultra high molecular-weight polyethylene fibres. J. Mech. Phys. Solids 63, 320-335. https://doi.org/10.1016/j.jmps.2013.08.021

Mayo, J.B., Wetzel, E.D., Hosur, M. V., Jeelani, S., 2009. Stab and puncture characterization of thermoplastic-impregnated aramid fabrics. Int. J. Impact Eng. 36, 1095-1105. https://doi.org/10.1016/j.ijimpeng.2009.03.006

Mines, R.A.W., Roach, A.M., Jones, N., 1999. High velocity perforation behaviour of polymer composite laminates. Int. J. Impact Eng. 22, 561-588. https://doi.org/10.1016/S0734743X(99)00019-6

Mitrevski, T., Marshall, I.H., Thomson, R., Jones, R., Whittingham, B., 2005. The effect of impactor shape on the impact response of composite laminates. Compos. Struct. 67, 139-148. https://doi.org/10.1016/j.compstruct.2004.09.007

Nazarian, O., Zok, F.W., 2014. Constitutive model for the shear response of Dyneema ${ }^{\circledR}$ fiber composites. Compos. Part A Appl. Sci. Manuf. 66, 73-81. https://doi.org/10.1016/j.compositesa.2014.06.012

O’Masta, M.R., Crayton, D.H., Deshpande, V.S., Wadley, H.N.G., 2016. Indentation of polyethylene laminates by a flat-bottomed cylindrical punch. Compos. Part A Appl. Sci. Manuf. 80, 138-147. https://doi.org/10.1016/j.compositesa.2015.10.015

O’Masta, M.R., Crayton, D.H., Deshpande, V.S., Wadley, H.N.G., 2015. Mechanisms of penetration in polyethylene reinforced cross-ply laminates. Int. J. Impact Eng. 86, 249-264. https://doi.org/10.1016/j.ijimpeng.2015.08.012

Onyechi, P.C., Edelugo, S.O., Chukwumuanya, E.O., Obuka, S.P.N., 2014. Ballistic Penetration Response Of Glass Fibre Reinforced Polyester ( Gfrp ) Composites : Body Amour 3, 226-237.

Phoenix, S.L., Porwal, P.K., 2003. A new membrane model for the ballistic impact response and V50 performance of multi-ply fibrous systems. Int. J. Solids Struct. 40, 6723-6765. https://doi.org/10.1016/S0020-7683(03)00329-9

Russell, B.P., Karthikeyan, K., Deshpande, V.S., Fleck, N.A., 2013. The high strain rate response of Ultra High Molecular-weight Polyethylene: From fibre to laminate. Int. J. Impact Eng. 60, 1-9. https://doi.org/10.1016/j.ijimpeng.2013.03.010

Shergold, O.A., Fleck, N.A., 2004. Mechanisms of deep penetration of soft solids, with application to the injection and wounding of skin. Proc. R. Soc. A Math. Phys. Eng. Sci. 460, 3037-3058. https://doi.org/10.1098/rspa.2004.1315 
Smith, P., Lemstra, P.J., 1980. Ultra-high strength polyethylene filaments by solution spinning/drawing. J Mater Sci 15, 505-514. https://doi.org/10.1016/0032-3861(80)90205-0

Smith, P., Lemstra, P.J., Kalb, B., Pennings, A.J., 1979. Ultrahigh-strength polyethylene filaments by solution spinning and hot drawing. Polym. Bull. 1, 733-736. https://doi.org/10.1007/BF00256272

van Dingenen, J., 1989. High performance dyneema fibres in composites. Mater. Des. 10, 101-104. https://doi.org/10.1016/S0261-3069(89)80021-4

Wen, H.M., 2000. Predicting the penetration and perforation of FRP laminates struck normally by projectiles with different nose shapes. Compos. Struct. 49, 321-329. https://doi.org/10.1016/S0263-8223(00)00064-7

Wilding, M.A., Ward, I.M., 1978. Tensile creep and recovery in ultra-high modulus linear polyethylenes. Polymer (Guildf). 19, 969-976. https://doi.org/10.1016/0032-3861(78)90208-2 